\title{
Risk of second breast cancer in female Hodgkin's lymphoma survivors: a meta-analysis
}

\author{
Ezzeldin M Ibrahim ${ }^{1 *+}$, Khaled M Abouelkhair ${ }^{1}$, Ghieth A Kazkaz' ${ }^{1}$ Osama A Elmasri ${ }^{1}$ and Meteb Al-Foheidi ${ }^{2+}$
}

\begin{abstract}
Background: Women treated for Hodgkin's lymphoma (HL) have an elevated risk of developing second breast cancer (SBC) compared with the general population. We planned this meta-analysis to quantify the long-term risk of SBC and analyze the contributing risk factors among HL survivors.

Methods: According to predefined selection criteria, literature search identified 34 studies that were included in the analyses.

Results: After eliminating overlapping or duplicate data, 957 incidences of SBC were encountered in 24,505 females with $\mathrm{HL}$ over a median follow-up of 14.9 years. The medians: age at the diagnosis of $\mathrm{HL}$, age at diagnosis of SBC, and latency since $\mathrm{HL}$ treatment to the development of SBC were 23.7, 35.0, and 17.7 years, respectively. The pooled relative risk (RR) of SBC was $8.23\left(95 \% \mathrm{Cl}, 5.43-12.47, \mathrm{P}^{2}=96 \%\right)$, with a median absolute excess rate of 22.9 per 10,000 person-years. The RR was found inversely related to age at diagnosis of $\mathrm{HL}$ with the highest rate $(68.7 ;[95 \% \mathrm{Cl}$, 28.08-168.11], $P^{2}=79 \%$ ), occurred in young patients ( $\leq 15$ years old), where the RR in older women ( $\geq 40$ years old) was not significant $(0.55 ;$ [ $95 \% \mathrm{Cl}, 0.09-3.52])$. Analysis of RR by 5 -year increments since the treatment of HL showed that the risk was highest after 15-19 years of latency $\left(13.87 ;[95 \% \mathrm{Cl}, 7.91-24.30], P^{2}=89 \%\right)$. Analysis of the effect of treatment modalities showed that the RR rates were $\left(4.70\right.$; [95\% Cl, 3.28-6.75], $\left.P^{2}=74 \%\right),(5.65$; [95\% $\mathrm{Cl}, 2.94-10.88]$, $P=91 \%)$, and (1.19; [95\% Cl, 0.50-2.82], $P=65 \%)$, for radiotherapy (RT) only, combined RT and chemotherapy (CT), and $C T$ only, respectively. To investigate the demonstrated heterogeneity, meta-regression analysis was performed when feasible. In most such analyses, the natural logarithm of RR was inversely associated with age at HL diagnosis.

Conclusions: We conclude that, the current meta-analysis provided the most recent comprehensive estimate of the risk of SBC in a broad-range of HL survivors. Younger age at diagnosis proved to be a dominant risk factor. The obtained results would serve providing breast cancer screening recommendations for HL survivors.
\end{abstract}

\section{Background}

Hodgkin's lymphoma (HL) became a curable disease by radiation therapy (RT) and/or combination chemotherapy (CT) since the early 70s [1-3]. Long-term diseasefree survival of $70 \%$ to $90 \%$, depending on stage at diagnosis has been achieved [4], and even more favorable outcome has been demonstrated in pediatrics, with a 5-year survival exceeding 90\% [5].

However, increased risk of second cancer following effective treatment of HL has long been reported [6]. More recently, second neoplasms after HL are being

\footnotetext{
* Correspondence: ezzibrahim@imc.med.sa

${ }^{\dagger}$ Equal contributors

${ }^{1}$ Oncology Center of Excellence, International Medical Center, PO Box 2172, Jeddah 21451, Saudi Arabia

Full list of author information is available at the end of the article
}

encountered with increasing frequency due to the marked improvement in survival [2,7]. The particular elevated risk of second breast cancer (SBC) among this population is not surprising in view of the reported excess risks of breast cancer $(\mathrm{BC})$ after incidental low doses of ionizing radiation $[8,9]$, therapeutic RT $[10,11]$, or as sequelae of the carcinogenic effects of CT [12,13].

Two pertinent meta-analyses have been published and they have addressed different questions. The first metaanalysis was published in 2006 and examined all second malignancy risk associated with HL treatment in 31 randomized trials and it included 65 incidence of SBC [14]. In the second meta-analysis [15], SBC risk and $\mathrm{BC}$ surveillance were investigated in young females $(\leq 30$ years at the primary tumor diagnosis) receiving moderate to high doses of RT targeted to mantle and modified

\section{Biomed Central}


mantle fields, mediastinum, lung, and thorax [15]. The latter meta-analysis comprised 11 studies that were not restricted to patients with HL but included all primary neoplasms in that age group.

Research on the late consequences of HL has often been limited by the size and composition of the study populations and by the duration and completeness of patient follow-up. To the best of our knowledge, there is no recently published meta-analysis intended to examine the risk of SBC in a broad range of ages at HL diagnosis, various follow-up periods, and subsequent to different therapeutic modalities. Also not precisely known, is the effect of other contributing risk factors. The lack of such data has prompted the current meta-analysis.

\section{Methods}

\section{Search strategy}

Between January 1966 and October 2011, we identified studies of interest by first conducting an electronic literature search of the databases MEDLINE, EMBASE, and the Cochrane Library. We also searched for relevant abstracts in the annual conference proceedings between January 1984 to October 2011 for the American Society of Clinical Oncology, European Society for Medical Oncology, and the San Antonio Breast Cancer Symposium. All ages of HL patients were eligible for inclusion.

We used exploded Medical Subject Heading terms or key words terms 'lymphoma, 'Hodgkin, 'Hodgkin's disease' and 'Hodgkin's lymphoma'. The terms were combined with 'neoplasm, second neoplasm, second primary' using the Boolean operator 'and'. Search results were also filtered against the terms 'breast, breast cancer, breast neoplasm). In the second step, these keywords were combined using the Boolean operator 'and' with 'standardized incidence ratio,' 'relative risk', and 'observed to expected'. In addition, we manually reviewed the reference lists of relevant studies to identify additional pertinent published articles.

\section{Selection criteria}

We included studies that met each of following criteria: (i) published in English language between January 1985 and October 2011; (ii) included naive patients at any age and with any stage of HL; (iii) investigated the risk for second malignant neoplasms (SMNs) in HL survivors; (iv) reported relative risk (RR) and/or specified as standardized incidence ratios (SIR) or data allowing such outcomes to be derived; and (v) published as original articles (no case reports, case series, reviews, comments, letters, or editorials).

When two or more references reported duplicate data, we only included in the analysis the most recent data, studies with the longer follow-up, or the most relevant studies. We excluded studies that mainly addressed the clinical characteristics of SBC. We also excluded studies that mainly intended to evaluate the potential benefits and harms associated with breast cancer surveillance among women with HL. Case-control designs, i.e. HL patients who developed $\mathrm{BC}$ compared with patients who did not were excluded.

\section{Data extraction}

Two authors (KMA, and GAK) independently inspected each reference title identified by the search and applied the inclusion criteria. For possibly relevant articles and in cases of disagreement between reviewers, the full article was obtained and inspected independently by the five authors. The data intended for extraction were discussed, and decisions were documented. We used the STROBE (Strengthening the Reporting of Observational Studies in Epidemiology) reporting criteria to assess the quality of studies included in the meta-analysis [16]. Any significant lack of concordance in the scores assigned by authors was discussed to reach a consensus.

Standardized Excel sheet was used for each study that fulfilled the inclusion criteria. Extracted data included paper characteristics (first author's last name, publication year, country in which the study was carried out, and data source), study design, number of HL patients, mean/median age of patients, mean/median duration of follow-up, therapy details, number of observed and expected SBC cases, and RR or standardized-incidence rate (SIR) with corresponding 95\% confidence interval (CI). The ratio of observed to expected numbers of cancers, SIR (referred to in the text as RR) was then used or calculated with likelihood-based 95\% CI from Poisson models [17]. Where not reported, we computed the CI for the risk assuming a Poisson distribution for the observed number of cases. Standard error (SE) for the natural logarithm of RR (lnRR) was derived from CI, applying the following equation: $\mathrm{SE}=\ln$ (upper $95 \% \mathrm{CI}$ / lower $95 \% \mathrm{CI}) /\left(2 \times \mathrm{z}_{1}-a / 2\right)$. When appropriate, we also used the built-in calculator of the Review Manager Software (version 5.1.4 for Windows; The Cochrane Collaboration, Oxford, UK) to compute missing data. When studies showed that the observed number of cases was zero, we simply added 1 to both the observed and the expected number of cases to allow computation of an estimate of the $\operatorname{lnRR}$ and its associated SE [18].

\section{Outcome measures}

The primary outcome was the overall pooled RR of incidence of SBC among women survivors of HD. The secondary end points were RR vs. various variables: source of data, age at diagnosis of HD, length of follow-up, treatment modalities, and any additional relevant risk factors. $\mathrm{RT}$ in this meta-analysis is referred to supra-diaphragmatic irradiation with or without other radiation fields. 


\section{Statistical analyses}

We assessed heterogeneity of the studies' results by inspecting graphical presentations and by calculating an $x^{2}$ test of heterogeneity and the $I^{2}$ statistic of inconsistency $[19,20]$. Statistically significant heterogeneity was defined as a $x 2 P$ value less than .1 or an $I 2$ statistic greater than $50 \%$. The estimates of RR, together with associated $95 \% \mathrm{CI}$, were obtained using the DerSimonian and Laird random-effects model [21]. Meta-regression analysis was performed to determine to what extent the heterogeneity is explained by various covariates using IBM SPSS statistical package v.19. The dependent variable was the $\ln R R$ weighted for the inverse of variance to perform weighted least square linear regression. We first conducted a univariate regression analysis for each variable followed by a multivariate regression including variables found significant in the univariate analysis.

Subgroup analyses were performed to assess potential contributions of various clinical variables to outcomes. A funnel plot estimating the precision of trials (plots of logarithm of the RR against the sample size) was examined for asymmetry to estimate publication bias [22]. Publication bias was also quantified by the regression asymmetry test by Egger [22].

All statistical tests were two-sided. RR was estimated according to the inverse of variance method with the use of Review Manager Software v5.1.4.

\section{Results}

\section{Search results}

We identified 1,647 potentially relevant articles (Figure 1). After exclusion of duplicate references, none-relevant literature, and those that did not satisfy inclusion criteria, 41 candidate articles were considered for the meta-analysis [23-62]. After careful review of the full text of these articles, 7 studies were excluded. In 5 studies the RR was reported based on case-control design, i.e. HL patients who developed SBC against patients who did not $[33,43,47,63,64]$. The RR in the sixth excluded study compared RR among HL patients according to presence or absence of a family history of BC [50]. The seventh excluded study was designed to examine RT dose and dose distribution in $41 \mathrm{HL}$ patients (25 females) treated at a single Canadian institution [49].

The remaining 34 studies were included in the metaanalysis. The funnel plot of 25 non-overlapping studies showed mild asymmetry, however, the Egger linear regression test was not significant $(P=.059)$.

Of the 34 included studies, there were several reports of overlapping and/or updated data with longer follow-up and more encountered events. For any analysis, only the updated results were used unless there were relevant data available in earlier report and were not included in more recent publication. That approach was used to deal with the overlapping data of van Leeuwen et al. [25] (2 Dutch institutes) and De Bruin et al. [55] (5 Dutch institutes); Mauch et al. [26] (5 USA institutes) and Ng et al. [41] (4 USA institutes); Aisenberg et al. [28] and Alm El-Din et al. [54] (single USA institute); Basu et al. [52] and Constine et al. [53] (5 USA institutes); Neglia et al. [39], Kenney et al. [45], and Castellino et al. [59] (the multiinstitutional USA Childhood Cancer Survivor Study); Metayer et al. [35] (16 population-based cancer registries in North America and Europe, Hodgson et al. [48] (13 population-based cancer registries in North America and Europe), and that of Dores et al. [57] (9 populationbased cancer registries in the USA); Hancock et al. [24], Wolden et al. (1998) [30], O'Brien [58], and Wolden et al. (2000) [37] (single USA institute); and Swerdlow et al. [36], and Swerdlow et al. [62] for the British National Lymphoma Investigation.

Tables 1 and 2 show the abstracted and computed data of the included studies. Overlapping studies were shaded together. The data sets included patients diagnosed as early as 1935 to the late 2000s. The median duration of follow-up since the diagnosis of HL was 14.9 years (95\% CI, 13.0-16.2 years; ranging from 6 to 23.8 years), while the median age at the diagnosis of HL was 23.7 years (95\% CI, 18.9-25.5 years; ranging from 11.8 to 40 years), and the median age at the diagnosis of SBC was 35.0 years (95\% CI, 30.2-40.0 years; ranging from 12.8 to 44.4 years). The median interval between primary diagnosis and development of SBC was 17.7 years (95\% CI, 15.2-18.6 years; ranging from 12.2 to 21.6 years).

After eliminating overlapping and duplicate studies, there were 25,305 women with HL and 957 incidences of SBC. The median absolute excess rate (AER) of SBC incidence per 10,000 person-years of observation was 22.9 excess cases $(95 \% \mathrm{CI}, 15.6-55.7$, ranging from 1.1 to 174). Few studies reported the cumulative incidence of SBC after 20 and 30 years of follow-up (average: $5.4 \%$ and 12\%, respectively).

\section{Pooled RR}

Figure 2 depicts the Forest plot for the pooled RR. The fixed-effects model showed significant heterogeneity $\left(I^{2}\right.$ statistic $\left.=96 \% ; \mathrm{p}<0.0001\right)$. The random-effects model was computed instead and it showed that patients with HL have an almost 9-fold increase in the risk of SBC ( RR = 8.23; [95\% CI, 5.43-12.47], $I^{2}=96 \%$ ). We performed meta-regression analysis to determine to what extent the heterogeneity is explained by the effects of study size, age at HL diagnosis, and the latency since the completion of HL treatment. The univariate analysis showed significant inverse association between $\ln R R$ and age at diagnosis and a positive relation to latency since HL treatment. With multivariate analysis (Table 3 ), only younger age at diagnosis remained significant $(<0.0001)$. 


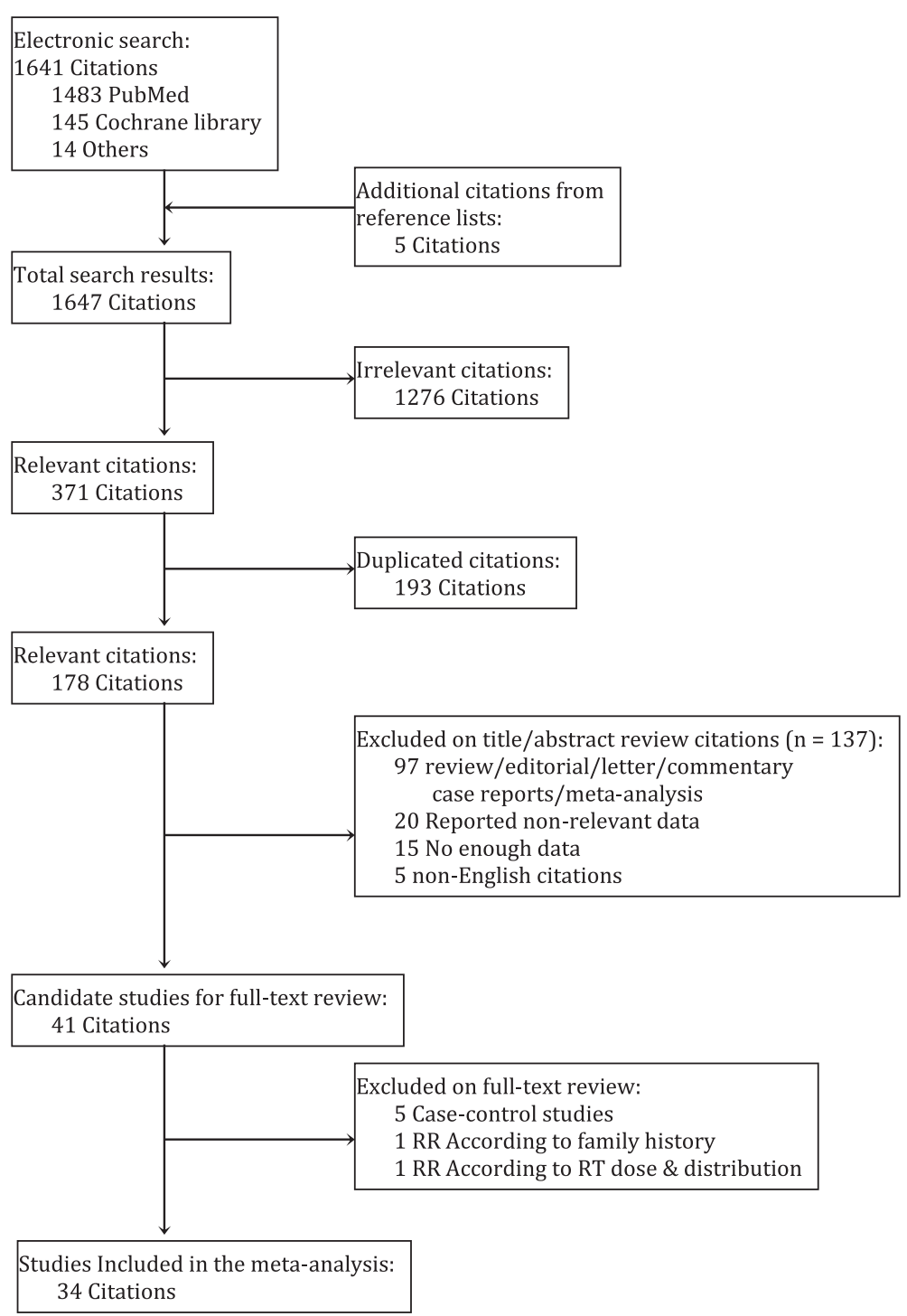

Figure 1 Search results and the selection of 33 included studies.

In Figure 3, the random-effects analysis illustrates a higher risk in institutional studies $(\mathrm{RR}=8.86$; [95\% CI, 5.26-14.94] compared with population-based analyses (6.70; [95\% CI, 4.07-11.03]). The demonstrated heterogeneity $\left(I^{2}=96 \%\right)$ was explored by meta-regression analysis, however, none of variables tested was found associated with $\ln R R$ in the univariate analysis (data not shown).

\section{$\mathrm{RR}$ and age at diagnosis of $\mathrm{HL}$}

The excess risk of SBC as a function of age at diagnosis of HL was also explored. Figure 4 (random-effects model) shows that the RR of developing SBC decreased significantly with increasing age at diagnosis from 68.7 (95\% CI, 28.8-168.11, $\leq 15$ years old) to 22.32 (95\% CI, 13.4-37.16, 15-19 years old), 14.43 (95\% CI, 11.65-17.88, 20-24 years old), and 6.6 (95\% CI, 4.24-10.29, 25-29 years old). As a significant heterogeneity was shown $\left(I^{2}=79 \%\right)$, we performed a meta-regression analysis, however, none of the explanatory variables was found significant (data not shown).

Several studies reported the RR at certain cut points of age at diagnosis and it also showed an inverse relation between risk and age (Figure 5, fixed-effects model). In the latter analysis and contrary to analysis of RR against more age intervals, no significant heterogeneity was noted $\left(I^{2}=41 \%\right)$. Of note, the RR of SBC for women who developed HL above the age of 40 years was not significant $(\mathrm{RR}=0.55$; [95\% CI, 0.09-3.52]).

\section{RR and follow-up latency}

We performed analysis of RR by 5 -year increments since the treatment of HL (Figure 6, random-effects model, $\left.I^{2}=73 \%\right)$. By and large, the analysis demonstrated an 
Table 1 Country, study period, design, source of data, study quality, and Hodgkin's lymphoma therapy details of the 34 studies included in the meta-analysis

\begin{tabular}{|c|c|c|c|c|c|c|c|c|c|}
\hline Author \& Year & Country & Year & Design & Source of data & $\begin{array}{c}\text { STROBE } \\
\text { criteria (met/ } \\
\text { applicable) }\end{array}$ & $\begin{array}{l}\text { RT only } \\
\text { No. }(\%)\end{array}$ & $\begin{array}{l}\text { CTX only } \\
\text { No. (\%) }\end{array}$ & $\begin{array}{l}\text { RT-CTX } \\
\text { No. }(\%)\end{array}$ & Comments \\
\hline Coleman 1987 [23] & UK & 1961-1980 & PBCS. & Cancer registry & $25 / 32$ & $(35-36)$ & $(20-28)$ & $(27-34)$ & $\begin{array}{l}\text { Therapy data for } M+F \\
\text { HL patients. Number NR. }\end{array}$ \\
\hline Hancock 1993 [24] & USA & 1961-1989 & $\begin{array}{l}\text { Single institution } \\
\text { cohort study }\end{array}$ & $\begin{array}{l}\text { Computerized database } \\
\text { and patient records }\end{array}$ & $24 / 31$ & $383(43)$ & $30(3)$ & $341(39)$ & $\begin{array}{l}\text { Therapy data for } \mathrm{M}+\mathrm{F} \mathrm{HL} \text { patients. } \\
15 \% \text { did not receive any treatment. }\end{array}$ \\
\hline Wolden 1998 [30] & USA & 1960-1995 & $\begin{array}{l}\text { Single institution cohort } \\
\text { study (HL Dx. } \leq 21 \mathrm{y})\end{array}$ & $\begin{array}{l}\text { Computerized database } \\
\text { and patient records }\end{array}$ & $22 / 31$ & $144(47)$ & $9(3)$ & $154(50)$ & Therapy data for female patients \\
\hline Wolden 2000 [37] & USA & 1960-1997 & $\begin{array}{l}\text { Single institution } \\
\text { retrospective review }\end{array}$ & $\begin{array}{l}\text { Computerized database } \\
\text { and patient records }\end{array}$ & $24 / 32$ & $37(57)$ & $1(2)$ & $27(41)$ & $\begin{array}{l}\text { Therapy data for SBC } \\
\text { patients. } 27 \text { patients } \\
\text { received alkylating CTX. }\end{array}$ \\
\hline O'Brien 2010 [58] & USA & 1970-1990 & $\begin{array}{l}\text { Single institution } \\
\text { study of children }\end{array}$ & $\begin{array}{l}\text { Retrospective chart review } \\
\text { and patient questionnaires }\end{array}$ & $23 / 31$ & $0(0)$ & $0(0)$ & $35(100)$ & $\begin{array}{l}\text { Therapy data for } \mathrm{M}+\mathrm{F} \\
\text { pediatric } \mathrm{HL} \text { patients. } \\
\text { All received low-dose RT } \\
\text { and alkylating CTX. }\end{array}$ \\
\hline $\begin{array}{l}\text { van Leeuwen } \\
1994[25]\end{array}$ & Netherlands & 1966-1986 & $\begin{array}{l}2 \text { institutions } \\
\text { cohort study }\end{array}$ & $\begin{array}{l}\text { Institutional registries } \\
\text { and patient records }\end{array}$ & $22 / 30$ & $552(29)$ & $178(9)$ & $1209(62)$ & $\begin{array}{l}\text { Therapy data for } M+F \\
\text { HL patients. All SBC received RT. }\end{array}$ \\
\hline De Bruin 2009 [55] & Netherlands & 1965-1995 & $\begin{array}{l}6 \text { institutions cohort } \\
\text { study (5y survivors) }\end{array}$ & $\begin{array}{l}\text { Medical records, and } \\
\text { physician questionnaires }\end{array}$ & $25 / 32$ & $357(31.8)$ & $80(7.1)$ & $685(61.1)$ & $\begin{array}{l}\text { Therapy data for all } \\
\text { HL female patients }\end{array}$ \\
\hline Mauch 1996 [26] & USA & 1969-1988 & $\begin{array}{l}5 \text { institutions } \\
\text { cohort study }\end{array}$ & Institutional records & $25 / 31$ & $489(62)$ & $0(0)$ & $305(38)$ & $\begin{array}{l}\text { Therapy data for } \mathrm{M}+\mathrm{F} \\
\mathrm{HL} \text { patients }\end{array}$ \\
\hline Ng 2002 [41] & USA & 1969-1997 & 4 institutions cohort study & Institutional records & $26 / 32$ & $665(69)$ & $0(0)$ & $296(31)$ & Therapy data for $\mathrm{M}+\mathrm{F} \mathrm{HL}$ patients \\
\hline Sankila 1996 [27] & Nordic countries & 1940-1987 & $\begin{array}{l}5 \text { Nordic PBCS } \\
\text { (HL Dx. } \leq 20 y)\end{array}$ & National cancer registries & $21 / 31$ & NR & NR & NR & All SBC patients received RT \\
\hline Metayer 2000 [36] & USA and Europe & 1935-1994 & $\begin{array}{l}16 \text { PBCS (HL Dx. } \leq 21 y \text {, } \\
1 \text {-year survivors) }\end{array}$ & National cancer registries & $22 / 32$ & $N R$ & NR & $N R$ & \\
\hline Hodgson 2007 [48] & USA and Europe & 1970-2001 & 13 PBCS (5-year survivors) & National cancer registries & $22 / 32$ & $\begin{array}{l}6461(34) \\
145(74)\end{array}$ & $\begin{array}{l}4398(23) \\
26(13)\end{array}$ & $\begin{array}{l}2847(15) \\
36(18)\end{array}$ & $\begin{array}{l}\text { First raw: therapy data for } M+F \\
\text { HL patients. ( } 27 \% \text { with unknown } \\
\text { treatment). Second raw:therapy } \\
\text { data for SBC patients ( } 28 \% \text { with } \\
\text { unknown treatment) }\end{array}$ \\
\hline Dores 2010 [57] & USA & $1973-2000$ & $\begin{array}{l}9 \text { PBCS (5-year survivors, } \\
\text { Dx. } \leq 35 y \text { ) }\end{array}$ & $\begin{array}{l}9 \text { cancer registry } \\
\text { areas of SEER }\end{array}$ & $22 / 32$ & NR & NR & NR & All patients had RT \\
\hline Aisenberg 1997 [28] & USA & 1964-1984 & $\begin{array}{l}\text { Single institution } \\
\text { cohort study }\end{array}$ & Review of patient records & $21 / 31$ & $10(71)$ & $0(0)$ & $4(29)$ & $\begin{array}{l}\text { Therapy data for SBC patients } \\
\text { ( } 4 \text { patients had alkylating CTX) }\end{array}$ \\
\hline Alm El-Din 2009 [54] & USA & $1964-2001$ & $\begin{array}{l}\text { Single institution } \\
\text { cohort study }\end{array}$ & Review of patient records & $21 / 31$ & $\begin{array}{l}130(52) \\
24(67)\end{array}$ & $\begin{array}{l}0(0) \\
0(0)\end{array}$ & $\begin{array}{l}118(48) \\
12(33)\end{array}$ & $\begin{array}{l}\text { First raw: therapy data for all } \\
\text { patients ( } 26 \% \text { had alkylating CTX) } \\
\text { Second raw: therapy data for SBC } \\
\text { ( } 22 \% \text { had alkylating CTX) }\end{array}$ \\
\hline Hudson 1998 [29] & USA & 1968-1990 & Single institution & Review of patient records & $21 / 30$ & $116(30)$ & $15(4)$ & $256(66)$ & Therapy data of M $+\mathrm{F} \mathrm{HL}$ patients \\
\hline $\begin{array}{l}\text { Gervais-Fagnou } \\
1999[31]\end{array}$ & Canada & 1965-1990 & $\begin{array}{l}\text { Single institution cohort } \\
\text { study (HL Dx. at } \leq 30 y \text { ) }\end{array}$ & Review of patient records & $22 / 30$ & $225(55)$ & $0(0)$ & $186(45)$ & Therapy data of M $+\mathrm{F} \mathrm{HL}$ patients \\
\hline
\end{tabular}


Table 1 Country, study period, design, source of data, study quality, and Hodgkin's lymphoma therapy details of the 34 studies included in the meta-analysis (Continued)

\begin{tabular}{|c|c|c|c|c|c|c|c|c|c|}
\hline Munker 1999 [32] & Germany & 1974-1994 & $\begin{array}{l}6 \text { institutions } \\
\text { cohort study }\end{array}$ & $\begin{array}{l}\text { Munich tumor registry, } \\
\text { patient records, and } \\
\text { patient \& family contact }\end{array}$ & $24 / 31$ & $484(43.1)$ & $169(15.1)$ & $464(41.4)$ & $\begin{array}{l}\text { Therapy data of } \mathrm{M}+\mathrm{F} \mathrm{HL} \text { patients } \\
\text { ( } 8 / 9 \mathrm{SBC} \text { patients received RT) }\end{array}$ \\
\hline Green 2000 [34] & USA & 1960-1989 & $\begin{array}{l}\text { Single institution cohort } \\
\text { study (HL } \leq 20 y \text { at Dx.) }\end{array}$ & $\begin{array}{l}\text { Patient records and } \\
\text { mail contact }\end{array}$ & $24 / 30$ & $1(25)$ & $0(0)$ & $3(75)$ & Therapy data for SBC patients \\
\hline Swerdlow 2000 [36] & United Kingdom & 1963-1993 & BNLI (cohort study) & BNLI + 2 cancer databases & $24 / 31$ & $1449(27)$ & $1693(31)$ & $2327(42)$ & $\begin{array}{l}\text { Therapy data of M+F HL patients } \\
\text { (all SBC had RT) }\end{array}$ \\
\hline Swerdlow 2011 [62] & United Kingdom & 1963-2001 & BNLI (cohort study) & $\begin{array}{l}\text { BNLI database } \\
\text { (70 institutions) }\end{array}$ & $25 / 31$ & $0(0)$ & $2366(41)$ & $3432(59)$ & $\begin{array}{l}\text { Therapy data of M+F HL patients } \\
\text { (SBC: } 14 \% \text { CTX only, } 86 \% \text { CTX + RT) }\end{array}$ \\
\hline Cellai 2001 [38] & Italy & 1960-1991 & $\begin{array}{l}\text { Single institution } \\
\text { cohort study }\end{array}$ & Institutional patient records & $22 / 30$ & $546(36)$ & $325(21)$ & $653(43)$ & $\begin{array}{l}\text { Therapy data of M+F HL patients } \\
\text { (SBC patients: } 6 \% \text { RT, } 94 \% \text { RT + CTX) }\end{array}$ \\
\hline Neglia 2001 [39] & USA and Canada & 1970-1986 & $\begin{array}{l}25 \text { institutions (CCSS) } \\
\text { cohort study } \\
\text { ( } \leq 21 \text { y at HL Dx., } \\
\text { 5-y survivors) }\end{array}$ & Institutional patient records & $26 / 32$ & $N R$ & NR & NR & $\begin{array}{l}\text { Therapy data were reported for all } \\
\text { children malignancies combined }\end{array}$ \\
\hline Kenney 2004 [45] & USA and Canada & 1970-1986 & Same as Neglia et al. [40] & Institutional patient records & $26 / 32$ & NR & NR & NR & $\begin{array}{l}\text { Therapy data were reported for all } \\
\text { children malignancies combined }\end{array}$ \\
\hline Castellino 2011 [59] & USA and Canada & 1970-1986 & $\begin{array}{l}\text { Same as Neglia et al. [40] } \\
\text { and Kenny et al. [46] }\end{array}$ & Institutional patient records & $26 / 32$ & $263(33)$ & $46(6)$ & $472(60)$ & $\begin{array}{l}\text { Therapy data for all } \\
\text { HL female patients }\end{array}$ \\
\hline $\begin{array}{l}\text { Foss Abrahamsen } \\
2002[40]\end{array}$ & Norway & 1968-1985 & $\begin{array}{l}\text { Single institution cohort } \\
\text { study (HD } \geq 1 y \text { survivors) }\end{array}$ & National cancer registry & $21 / 30$ & $447(44)$ & $202(20)$ & $363(36)$ & Therapy data of $\mathrm{M}+\mathrm{F} \mathrm{HL}$ patients \\
\hline Bhatia 2003 [42] & USA and Europe & 1955-1986 & $\begin{array}{l}15 \text { institutions } \\
\text { cohort study }\end{array}$ & Institutional patient records & $26 / 33$ & $\begin{array}{l}314(23) \\
14(47)\end{array}$ & $\begin{array}{l}106(8) \\
0(0)\end{array}$ & $\begin{array}{l}960(69) \\
15(53)\end{array}$ & $\begin{array}{l}\text { First raw: therapy data of } M+F \\
\text { HL patients Second raw: therapy } \\
\text { data of SBC patients }\end{array}$ \\
\hline $\begin{array}{l}\text { Wahner-Roedler } \\
2003 \text { [44] }\end{array}$ & USA & 1950-1993 & $\begin{array}{l}\text { Single institution } \\
\text { cohort study }\end{array}$ & Institutional patient records & $23 / 31$ & $\begin{array}{l}322(50) \\
23(77)\end{array}$ & $\begin{array}{l}0(0) \\
0(0)\end{array}$ & $\begin{array}{l}327(50) \\
7(23)\end{array}$ & $\begin{array}{l}\text { First raw: therapy data of } M+F \\
\text { HL patients Second raw: therapy } \\
\text { data of SBC patients }\end{array}$ \\
\hline Behringer 2004 [60] & Germany & 1981-1989 & $\begin{array}{l}\text { Multi-Institutional } \\
\text { cohort study }\end{array}$ & German HL database & $23 / 31$ & $675(12.9)$ & $618(11.8)$ & $3947(75.3)$ & Therapy data of $\mathrm{M}+\mathrm{F} \mathrm{HL}$ patients \\
\hline Guibout 2005 [46] & UK and France & 1954-1985 & 8 institutions cohort study & Institutional patient records & $28 / 32$ & $28(23)$ & $9(7)$ & $86(70)$ & Therapy data of $\mathrm{M}+\mathrm{F} \mathrm{HL}$ patients \\
\hline Taylor 2007 [51] & UK & 1940-1991 & PBCS & $\begin{array}{l}\text { National Registry of } \\
\text { Childhood Tumors }\end{array}$ & $22 / 31$ & $\begin{array}{l}121(37) \\
7(44)\end{array}$ & $\begin{array}{l}63(20) \\
0(0)\end{array}$ & $\begin{array}{l}138(43) \\
9(56)\end{array}$ & $\begin{array}{l}\text { First raw: therapy data of female } \\
\text { HL patients Second raw: therapy } \\
\text { data of SBC patients }\end{array}$ \\
\hline $\begin{array}{l}\text { Basu } 2008 \text { [52] } \\
\text { Constine } 2008 \text { [53] }\end{array}$ & USA & 1960-1990 & $\begin{array}{l}5 \text { institutions cohort } \\
\text { study }(<19 y \text { at HL Dx.) }\end{array}$ & Institutional patient records & $22 / 31$ & $\begin{array}{l}174(44) \\
18(62)\end{array}$ & $\begin{array}{l}37(9) \\
3(10)\end{array}$ & $\begin{array}{l}187(47) \\
8(28)\end{array}$ & $\begin{array}{l}\text { First raw: therapy data of } \mathrm{M}+\mathrm{F} \\
\text { HL patients Second raw: therapy } \\
\text { data of SBC patients }\end{array}$ \\
\hline Howell 2009 [56] & UK & 1965-2008 & $\begin{array}{l}\text { Cohort from a registry } \\
\text { and single institution }\end{array}$ & $\begin{array}{l}\text { Institutional patient } \\
\text { records and a registry data }\end{array}$ & $27 / 31$ & $6(26)$ & $0(0)$ & $17(74)$ & Therapy data of SBC patients \\
\hline Inskip 2007 [61] & USA & 1973-2002 & PBCS $(<18 y$ at Dx $)$ & SEER database & $29 / 31$ & NR & NR & NR & \\
\hline
\end{tabular}

BNLI British National Lymphoma Investigation, CCSS Childhood Cancer Survivors Study, Dx. diagnosis, HL Hodgkin's lymphoma, $M+F$ males and females, NR not reported or data could not be calculated, PBCS

population-based cohort study, RT radiotherapy that included supra-diaphragmatic irradiation, SBC second breast cancer, SEER Surveillance Epidemiology and End Results, STROBE Strengthening the Reporting of

Observational Studies in Epidemiology, y year. (studies shaded together represent overlapping data). 
Table $\mathbf{2}$ Clinical characteristics of the $\mathbf{3 4}$ studies included in the meta-analysis

\begin{tabular}{|c|c|c|c|c|c|c|c|c|c|}
\hline Author \& Year & Country & Year & Design & Source of data & $\begin{array}{l}\text { STROBE } \\
\text { criteria (met/ } \\
\text { applicable) }\end{array}$ & $\begin{array}{l}\text { RT only } \\
\text { No. (\%) }\end{array}$ & $\begin{array}{l}\text { CTX only } \\
\text { No. (\%) }\end{array}$ & $\begin{array}{l}\text { RT-CTX } \\
\text { No. (\%) }\end{array}$ & Comments \\
\hline Coleman 1987 [23] & UK & 1961-1980 & PBCS. & Cancer registry & $25 / 32$ & $(35-36)$ & $(20-28)$ & $(27-34)$ & $\begin{array}{l}\text { Therapy data for } \mathrm{M}+\mathrm{F} \\
\mathrm{HL} \text { patients. Number NR. }\end{array}$ \\
\hline Hancock 1993 [24] & USA & 1961-1989 & $\begin{array}{l}\text { Single institution } \\
\text { cohort study }\end{array}$ & $\begin{array}{l}\text { Computerized database } \\
\text { and patient records }\end{array}$ & $24 / 31$ & $383(43)$ & $30(3)$ & $341(39)$ & $\begin{array}{l}\text { Therapy data for } M+F \\
\text { HL patients. } 15 \% \text { did not } \\
\text { receive any treatment. }\end{array}$ \\
\hline Wolden 1998 [30] & USA & 1960-1995 & $\begin{array}{l}\text { Single institution } \\
\text { cohort study } \\
\text { (HL Dx. } \leq 21 y \text { ) }\end{array}$ & $\begin{array}{l}\text { Computerized database } \\
\text { and patient records }\end{array}$ & $22 / 31$ & $144(47)$ & $9(3)$ & $154(50)$ & $\begin{array}{l}\text { Therapy data for } \\
\text { female patients }\end{array}$ \\
\hline Wolden 2000 [37] & USA & 1960-1997 & $\begin{array}{l}\text { Single institution } \\
\text { retrospective review }\end{array}$ & $\begin{array}{l}\text { Computerized database } \\
\text { and patient records }\end{array}$ & $24 / 32$ & $37(57)$ & $1(2)$ & $27(41)$ & $\begin{array}{l}\text { Therapy data for SBC patients. } \\
27 \text { patients received } \\
\text { alkylating CTX. }\end{array}$ \\
\hline O'Brien 2010 [58] & USA & 1970-1990 & $\begin{array}{l}\text { Single institution } \\
\text { study of children }\end{array}$ & $\begin{array}{l}\text { Retrospective chart review } \\
\text { and patient questionnaires }\end{array}$ & $23 / 31$ & $0(0)$ & $0(0)$ & $35(100)$ & $\begin{array}{l}\text { Therapy data for } \mathrm{M}+\mathrm{F} \\
\text { pediatric HL patients. } \\
\text { All received low-dose } \\
\text { RT and alkylating CTX. }\end{array}$ \\
\hline $\begin{array}{l}\text { van Leeuwen } \\
1994[25]\end{array}$ & Netherlands & 1966-1986 & $\begin{array}{l}2 \text { institutions } \\
\text { cohort study }\end{array}$ & $\begin{array}{l}\text { Institutional registries } \\
\text { and patient records }\end{array}$ & $22 / 30$ & $552(29)$ & $178(9)$ & $1209(62)$ & $\begin{array}{l}\text { Therapy data for } \mathrm{M}+\mathrm{F} \\
\mathrm{HL} \text { patients. } \\
\text { All SBC received RT. }\end{array}$ \\
\hline De Bruin 2009 [55] & Netherlands & 1965-1995 & $\begin{array}{l}6 \text { institutions } \\
\text { cohort study } \\
\text { (5y survivors) }\end{array}$ & $\begin{array}{l}\text { Medical records, } \\
\text { and physician questionnaires }\end{array}$ & $25 / 32$ & $357(31.8)$ & $80(7.1)$ & $685(61.1)$ & $\begin{array}{l}\text { Therapy data for all } \\
\text { HL female patients }\end{array}$ \\
\hline Mauch 1996 [26] & USA & 1969-1988 & $\begin{array}{l}5 \text { institutions } \\
\text { cohort study }\end{array}$ & Institutional records & $25 / 31$ & $489(62)$ & $0(0)$ & $305(38)$ & $\begin{array}{l}\text { Therapy data for } \mathrm{M}+\mathrm{F} \\
\mathrm{HL} \text { patients }\end{array}$ \\
\hline Ng 2002 [41] & USA & 1969-1997 & $\begin{array}{l}4 \text { institutions } \\
\text { cohort study }\end{array}$ & Institutional records & $26 / 32$ & $665(69)$ & $0(0)$ & $296(31)$ & $\begin{array}{l}\text { Therapy data for } \mathrm{M}+\mathrm{F} \\
\mathrm{HL} \text { patients }\end{array}$ \\
\hline Sankila 1996 [27] & Nordic countries & 1940-1987 & $\begin{array}{l}5 \text { Nordic PBCS } \\
(\text { HL Dx. } \leq 20 y)\end{array}$ & National cancer registries & $21 / 31$ & NR & NR & NR & All SBC patients received RT \\
\hline Metayer 2000 [36] & USA and Europe & 1935-1994 & $\begin{array}{l}16 \text { PBCS (HL Dx. } \\
\leq 21 y, 1 \text {-year } \\
\text { survivors) }\end{array}$ & National cancer registries & $22 / 32$ & NR & NR & NR & \\
\hline Hodgson 2007 [48] & USA and Europe & 1970-2001 & $\begin{array}{l}13 \text { PBCS } \\
\text { (5-year survivors) }\end{array}$ & National cancer registries & $22 / 32$ & $\begin{array}{l}6461(34) \\
145(74)\end{array}$ & $\begin{array}{l}4398(23) \\
26(13)\end{array}$ & $\begin{array}{l}2847(15) \\
36(18)\end{array}$ & $\begin{array}{l}\text { First raw: therapy data for } \\
M+F \text { HL patients. } \\
\text { ( } 27 \% \text { with unknown } \\
\text { treatment). Second } \\
\text { raw:therapy data for } \\
\text { SBC patients ( } 28 \% \text { with } \\
\text { unknown treatment) }\end{array}$ \\
\hline Dores 2010 [57] & USA & 1973-2000 & $\begin{array}{l}9 \text { PBCS (5-year } \\
\text { survivors, Dx. } \leq 35 y)\end{array}$ & $\begin{array}{l}9 \text { cancer registry } \\
\text { areas of SEER }\end{array}$ & $22 / 32$ & NR & NR & NR & All patients had RT \\
\hline Aisenberg 1997 [28] & USA & 1964-1984 & $\begin{array}{l}\text { Single institution } \\
\text { cohort study }\end{array}$ & Review of patient records & $21 / 31$ & $10(71)$ & $0(0)$ & $4(29)$ & $\begin{array}{l}\text { Therapy data for SBC patients } \\
\text { (4 patients had alkylating CTX) }\end{array}$ \\
\hline
\end{tabular}


Table 2 Clinical characteristics of the $\mathbf{3 4}$ studies included in the meta-analysis (Continued)

\begin{tabular}{|c|c|c|c|c|c|c|c|c|c|}
\hline Alm El-Din 2009 [54] & USA & $1964-2001$ & $\begin{array}{l}\text { Single institution } \\
\text { cohort study }\end{array}$ & Review of patient records & $21 / 31$ & $\begin{array}{l}130(52) \\
24(67)\end{array}$ & $\begin{array}{l}0(0) \\
0(0)\end{array}$ & $\begin{array}{l}118(48) \\
12(33)\end{array}$ & $\begin{array}{l}\text { First raw: therapy data for all } \\
\text { patients ( } 26 \% \text { had alkylating } \\
\text { CTX) Second raw: } \\
\text { therapy data for SBC ( } 22 \% \\
\text { had alkylating CTX) }\end{array}$ \\
\hline Hudson 1998 [29] & USA & 1968-1990 & Single institution & Review of patient records & $21 / 30$ & $116(30)$ & $15(4)$ & $256(66)$ & $\begin{array}{l}\text { Therapy data of } M+F \\
H L \text { patients }\end{array}$ \\
\hline $\begin{array}{l}\text { Gervais-Fagnou } \\
1999 \text { [31] }\end{array}$ & Canada & 1965-1990 & $\begin{array}{l}\text { Single institution } \\
\text { cohort study } \\
\text { (HL Dx. at } \leq 30 y)\end{array}$ & Review of patient records & $22 / 30$ & $225(55)$ & $0(0)$ & $186(45)$ & $\begin{array}{l}\text { Therapy data of } M+F \\
\text { HL patients }\end{array}$ \\
\hline Munker 1999 [32] & Germany & 1974-1994 & $\begin{array}{l}6 \text { institutions } \\
\text { cohort study }\end{array}$ & $\begin{array}{l}\text { Munich tumor registry, patient } \\
\text { records, and patient } \\
\& \text { family contact }\end{array}$ & $24 / 31$ & $484(43.1)$ & $169(15.1)$ & $464(41.4)$ & $\begin{array}{l}\text { Therapy data of } \\
\mathrm{M}+\mathrm{F} \text { HL patients } \\
\text { ( } 8 / 9 \mathrm{SBC} \text { patients received RT) }\end{array}$ \\
\hline Green 2000 [34] & USA & 1960-1989 & $\begin{array}{l}\text { Single institution } \\
\text { cohort study } \\
\text { (HL } \leq 20 y \text { at Dx.) }\end{array}$ & $\begin{array}{l}\text { Patient records and } \\
\text { mail contact }\end{array}$ & $24 / 30$ & $1(25)$ & $0(0)$ & $3(75)$ & Therapy data for SBC patients \\
\hline Swerdlow 2000 [36] & United Kingdom & 1963-1993 & BNLI (cohort study) & BNLI + 2 cancer databases & $24 / 31$ & $1449(27)$ & $1693(31)$ & $2327(42)$ & $\begin{array}{l}\text { Therapy data of } M+F \\
\text { HL patients } \\
\text { (all SBC had RT) }\end{array}$ \\
\hline Swerdlow 2011 [62] & United Kingdom & $1963-2001$ & BNLI (cohort study) & BNLI database (70 institutions) & $25 / 31$ & $0(0)$ & $2366(41)$ & $3432(59)$ & $\begin{array}{l}\text { Therapy data of M+F } \\
\text { HL patients } \\
\text { (SBC: } 14 \% \text { CTX only, } \\
86 \% \text { CTX + RT) }\end{array}$ \\
\hline Cellai 2001 [38] & Italy & 1960-1991 & $\begin{array}{l}\text { Single institution } \\
\text { cohort study }\end{array}$ & Institutional patient records & $22 / 30$ & $546(36)$ & $325(21)$ & $653(43)$ & $\begin{array}{l}\text { Therapy data of } M+F \\
\text { HL patients } \\
\text { (SBC patients: } 6 \% \text { RT, } \\
94 \% \text { RT }+ \text { CTX) }\end{array}$ \\
\hline Neglia 2001 [39] & USA and Canada & 1970-1986 & $\begin{array}{l}25 \text { institutions } \\
\text { (CCSS) cohort } \\
\text { study ( } \leq 21 y \\
\text { at HL Dx., } \\
\text { 5-y survivors) }\end{array}$ & Institutional patient records & $26 / 32$ & NR & NR & NR & $\begin{array}{l}\text { Therapy data were reported } \\
\text { for all children } \\
\text { malignancies combined }\end{array}$ \\
\hline Kenney 2004 [45] & USA and Canada & 1970-1986 & $\begin{array}{l}\text { Same as Neglia } \\
\text { et al. [40] }\end{array}$ & Institutional patient records & $26 / 32$ & NR & NR & NR & $\begin{array}{l}\text { Therapy data were reported } \\
\text { for all children } \\
\text { malignancies combined }\end{array}$ \\
\hline Castellino 2011 [59] & USA and Canada & 1970-1986 & $\begin{array}{l}\text { Same as Neglia } \\
\text { et al. [40] and } \\
\text { Kenny et al. [46] }\end{array}$ & Institutional patient records & $26 / 32$ & $263(33)$ & $46(6)$ & $472(60)$ & $\begin{array}{l}\text { Therapy data for all } \\
\text { HL female patients }\end{array}$ \\
\hline $\begin{array}{l}\text { Foss Abrahamsen } \\
2002 \text { [40] }\end{array}$ & Norway & 1968-1985 & $\begin{array}{l}\text { Single institution } \\
\text { cohort study } \\
\text { (HD } \geq 1 \text { y survivors) }\end{array}$ & National cancer registry & $21 / 30$ & $447(44)$ & $202(20)$ & $363(36)$ & $\begin{array}{l}\text { Therapy data of } M+F \\
\mathrm{HL} \text { patients }\end{array}$ \\
\hline Bhatia 2003 [42] & USA and Europe & 1955-1986 & $\begin{array}{l}15 \text { institutions } \\
\text { cohort study }\end{array}$ & Institutional patient records & $26 / 33$ & $\begin{array}{c}314(23) \\
14(47)\end{array}$ & $\begin{array}{l}106(8) \\
0(0)\end{array}$ & $\begin{array}{c}960(69) \\
15(53)\end{array}$ & $\begin{array}{l}\text { First raw: therapy data } \\
\text { of } \mathrm{M}+\mathrm{F} H \mathrm{HL} \text { patients }\end{array}$ \\
\hline
\end{tabular}


Table 2 Clinical characteristics of the $\mathbf{3 4}$ studies included in the meta-analysis (Continued)

\begin{tabular}{|c|c|c|c|c|c|c|c|c|c|}
\hline & & & & & & & & & $\begin{array}{l}\text { Second raw: therapy } \\
\text { data of SBC patients }\end{array}$ \\
\hline $\begin{array}{l}\text { Wahner-Roedler } \\
2003 \text { [44] }\end{array}$ & USA & 1950-1993 & $\begin{array}{l}\text { Single institution } \\
\text { cohort study }\end{array}$ & Institutional patient records & $23 / 31$ & $\begin{array}{l}322(50) \\
23(77)\end{array}$ & $\begin{array}{l}0(0) \\
0(0)\end{array}$ & $\begin{array}{l}327(50) \\
7(23)\end{array}$ & $\begin{array}{l}\text { First raw: therapy data } \\
\text { of } \mathrm{M}+\mathrm{F} \mathrm{HL} \text { patients } \\
\text { Second raw: therapy data } \\
\text { of SBC patients }\end{array}$ \\
\hline Behringer 2004 [60] & Germany & 1981-1989 & $\begin{array}{l}\text { Multi-Institutional } \\
\text { cohort study }\end{array}$ & German HL database & $23 / 31$ & $675(12.9)$ & $618(11.8)$ & $3947(75.3)$ & $\begin{array}{l}\text { Therapy data of } \mathrm{M}+\mathrm{F} \\
\mathrm{HL} \text { patients }\end{array}$ \\
\hline Guibout 2005 [46] & UK and France & 1954-1985 & $\begin{array}{l}8 \text { institutions } \\
\text { cohort study }\end{array}$ & Institutional patient records & $28 / 32$ & $28(23)$ & $9(7)$ & $86(70)$ & $\begin{array}{l}\text { Therapy data of } \mathrm{M}+\mathrm{F} \\
\mathrm{HL} \text { patients }\end{array}$ \\
\hline Taylor 2007 [51] & UK & 1940-1991 & PBCS & $\begin{array}{l}\text { National Registry of } \\
\text { Childhood Tumors }\end{array}$ & $22 / 31$ & $\begin{array}{l}121(37) \\
7(44)\end{array}$ & $\begin{array}{c}63(20) \\
0(0)\end{array}$ & $\begin{array}{l}138(43) \\
9(56)\end{array}$ & $\begin{array}{l}\text { First raw: therapy data of } \\
\text { female HL patients } \\
\text { Second raw: therapy data of } \\
\text { SBC patients }\end{array}$ \\
\hline $\begin{array}{l}\text { Basu } 2008 \text { [52] } \\
\text { Constine } 2008 \text { [53] }\end{array}$ & USA & 1960-1990 & $\begin{array}{l}5 \text { institutions } \\
\text { cohort study } \\
\text { (<19y at HL Dx.) }\end{array}$ & Institutional patient records & $22 / 31$ & $\begin{array}{l}174(44) \\
18(62)\end{array}$ & $\begin{array}{l}37(9) \\
3(10)\end{array}$ & $\begin{array}{l}187(47) \\
8(28)\end{array}$ & $\begin{array}{l}\text { First raw: therapy data } \\
\text { of } \mathrm{M}+\mathrm{F} \mathrm{HL} \text { patients } \\
\text { Second raw: therapy data of } \\
\text { SBC patients }\end{array}$ \\
\hline Howell 2009 [56] & UK & $1965-2008$ & $\begin{array}{l}\text { Cohort from a } \\
\text { registry and } \\
\text { single institution }\end{array}$ & $\begin{array}{l}\text { Institutional patient } \\
\text { records and a registry data }\end{array}$ & $27 / 31$ & $6(26)$ & $0(0)$ & $17(74)$ & Therapy data of SBC patients \\
\hline Inskip 2007 [61] & USA & 1973-2002 & PBCS $(<18 y$ at Dx $)$ & SEER database & $29 / 31$ & NR & NR & NR & \\
\hline
\end{tabular}




\begin{tabular}{|c|c|c|c|c|c|c|c|c|c|}
\hline \multirow{2}{*}{$\begin{array}{l}\text { Study or Subgroup } \\
\text { Coleman } 1987\end{array}$} & \multirow{2}{*}{$\frac{\log [\text { Risk Ratio] }}{-0.69}$} & \multirow{2}{*}{$\begin{array}{r}\text { SE } \\
0.691\end{array}$} & \multirow{2}{*}{$\frac{\text { Weight }}{3.1 \%}$} & \multirow{2}{*}{\begin{tabular}{c}
\multicolumn{1}{c}{$\begin{array}{l}\text { Risk Ratio } \\
\text { IV, } \text { Random, 95\% CI }\end{array}$} \\
$0.50[0.13,1.94]$
\end{tabular}} & Year & \multicolumn{4}{|c|}{$\begin{array}{c}\text { Risk Ratio } \\
\text { IV, Random, } 95 \% \mathrm{Cl}\end{array}$} \\
\hline & & & & & 1987 & & & 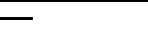 & \\
\hline Sankila 1996 & 2.83 & 0.265 & $4.3 \%$ & $16.95[10.08,28.49]$ & 1996 & & & & \\
\hline Wolden 1998 & 3.27 & 0.267 & $4.3 \%$ & $26.31[15.59,44.40]$ & 1998 & & & & - \\
\hline Hudson 1998 & 3.5 & 0.456 & $3.8 \%$ & $33.12[13.55,80.94]$ & 1998 & & & & \\
\hline Munker 1999 & 0.88 & 0.299 & $4.2 \%$ & $2.41[1.34,4.33]$ & 1999 & & & $\longrightarrow$ & \\
\hline Gervais-Fagnou 1999 & 2.36 & 0.274 & $4.3 \%$ & $10.59[6.19,18.12]$ & 1999 & & & & \\
\hline Wolden 2000 & 1.55 & 0.145 & $4.5 \%$ & $4.71[3.55,6.26]$ & 2000 & & & - & \\
\hline Green 2000 & 2.05 & 0.571 & $3.5 \%$ & $7.77[2.54,23.79]$ & 2000 & & & & \\
\hline Swerdlow 2000 & 0.34 & 0.216 & $4.4 \%$ & $1.40[0.92,2.15]$ & 2000 & & & - & \\
\hline Cellai 2001 & 0.72 & 0.266 & $4.3 \%$ & $2.05[1.22,3.46]$ & 2001 & & & - & \\
\hline Foss Abrahamsen 2002 & 1.34 & 0.225 & $4.4 \%$ & $3.82[2.46,5.94]$ & 2002 & & & $\longrightarrow$ & \\
\hline $\mathrm{Ng} 2002$ & 1.9 & 0.17 & $4.5 \%$ & $6.69[4.79,9.33]$ & 2002 & & & $\mp$ & \\
\hline Wahner-Roedler 2003 & 1.06 & 0.189 & $4.5 \%$ & $2.89[1.99,4.18]$ & 2003 & & & - & \\
\hline Bhatia 2003 & 4.02 & 0.167 & $4.5 \%$ & $55.70[40.15,77.27]$ & 2003 & & & & - \\
\hline Behringer 2004 & 0.64 & 0.486 & $3.7 \%$ & $1.90[0.73,4.92]$ & 2004 & & & & \\
\hline Guibout 2005 & 4.26 & 0.512 & $3.7 \%$ & $70.81[25.96,193.16]$ & 2005 & & & & $\rightarrow$ \\
\hline Hodgson 2007 & 1.81 & 0.123 & $4.6 \%$ & $6.11[4.80,7.78]$ & 2007 & & & - & \\
\hline Taylor 2007 & 2.44 & 0.264 & $4.3 \%$ & $11.47[6.84,19.25]$ & 2007 & & & & \\
\hline Basu 2008 & 3.62 & 0.195 & $4.5 \%$ & $37.34[25.48,54.72]$ & 2008 & & & & 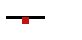 \\
\hline De Bruin 2009 & 1.72 & 0.1 & $4.6 \%$ & $5.58[4.59,6.79]$ & 2009 & & & - & \\
\hline Alm El-Din 2009 & 2.28 & 0.347 & $4.1 \%$ & $9.78[4.95,19.30]$ & 2009 & & & & \\
\hline Howell 2009 & 1.06 & 0.627 & $3.3 \%$ & $2.89[0.84,9.86]$ & 2009 & & & & \\
\hline O'Brien 2010 & 4.28 & 0.454 & $3.8 \%$ & $72.24[29.67,175.88]$ & 2010 & & & & $\rightarrow$ \\
\hline Castellino 2011 & 2.83 & 0.112 & $4.6 \%$ & $16.95[13.61,21.11]$ & 2011 & & & & \\
\hline \multicolumn{3}{|l|}{ Total $(95 \% \mathrm{Cl})$} & $100.0 \%$ & $8.23[5.43,12.47]$ & & & & & \\
\hline \multicolumn{6}{|c|}{$\begin{array}{l}\text { Heterogeneity: } \mathrm{Tau}^{2}=0.97 ; \mathrm{Chi}^{2}=530.68, \mathrm{df}=23(P<0.00001) ; \mathrm{I}^{2}=96 \% \\
\text { Test for overall effect: } Z=9.94(P<0.00001)\end{array}$} & 0.01 & 0.1 & 10 & 100 \\
\hline
\end{tabular}

increasing RR by increased duration of follow-up latency reaching the highest after $15-19$ years $(R R=13.87$; [95\% CI, 7.91-24.30]). While there was a decrease in RR noted after 20-24 years of follow-up, further rise occurred after 25-29 years. The latter rise may be attributed to the $R R$ reported by De Bruin et al. [55], while all the other studies demonstrated a decreased RR after 2529 years compared with that after 20-24 years of followup. Due to unreported data, meta-regression analysis of the heterogeneity could only include age at diagnosis of HL as the sole explanatory variable and it showed an inverse association with $\ln R R$ (Table 3 ).

After $\geq 20$ years of latency since diagnosis, 9 studies $[37,40,41,44,45,48,51,54,55]$ reported RR of 6.95 (95\% CI,
4.8- 10.1). That $R R$ was not significantly different from the rate encountered after $\geq 30$ years of followup $(\mathrm{RR}=7.03$; [95\% CI, 5.2-9.5]) as reported from 5 studies $[42,44,51,54,55]$.

\section{RR vs. Age at HL diagnosis and follow-up latency}

To examine the interaction of both age at HL diagnosis and length of follow-up versus risk, few studies have reported adequate data. De Bruin et al. [55] reported that after 5-14 years of follow-up, those who were $\leq 20$ years at $\mathrm{HL}$ diagnosis had significantly higher risk $(R R=20.0$; [95\% CI, 7.3-43.4]) as compared with those who were older ( $21-30$ years old) $(R R=5.3$; [95\% CI, 1.9- 16.6]). Similarly, after $\geq 25$ years of follow-up,

Table 3 The results of meta-regression analyses

\begin{tabular}{|c|c|c|c|c|}
\hline Model & Covariates & Meta-regression $\beta$ coefficient (SE) & $95 \% \mathrm{Cl}$ of $\beta$ coefficient & $p$ value \\
\hline $\begin{array}{l}\text { Pooled RR for included } \\
\text { studies (Figure 2) }\end{array}$ & $\begin{array}{l}\text { Age at } \mathrm{HL} \text { diagnosis } \\
\text { Latency since } \mathrm{HL} \text { treatment }\end{array}$ & $\begin{array}{l}-0.105(0.015) \\
-0.010(0.031)\end{array}$ & $\begin{array}{l}-0.137 \text { to }-0.072 \\
-0.075 \text { to } 0.055\end{array}$ & $<0.00010 .747$ \\
\hline $\begin{array}{l}\text { RR versus follow-up } \\
\text { intervals (Figure 6) }\end{array}$ & Age at $H L$ diagnosis & $-0.036(0.010)$ & -0.057 to -0.015 & 0.001 \\
\hline $\begin{array}{l}\text { RR versus therapy modality } \\
\text { (Figure } 7) \text { : RT vs. RT + C vs. C }\end{array}$ & $\begin{array}{l}\text { Age at } \mathrm{HL} \text { diagnosis } \\
\text { Latency since } \mathrm{HL} \text { treatment }\end{array}$ & $\begin{array}{l}-0.099(0.027) \\
-0.095(0.073)\end{array}$ & $\begin{array}{l}-0.157 \text { to }-0.041 \\
-0.251 \text { to } 0.061\end{array}$ & 0.0030 .212 \\
\hline
\end{tabular}

C chemotherapy, Cl confidence interval, HL Hodgkin's lymphoma, RR relative risk, $R T$ radiotherapy, SE standard error. 


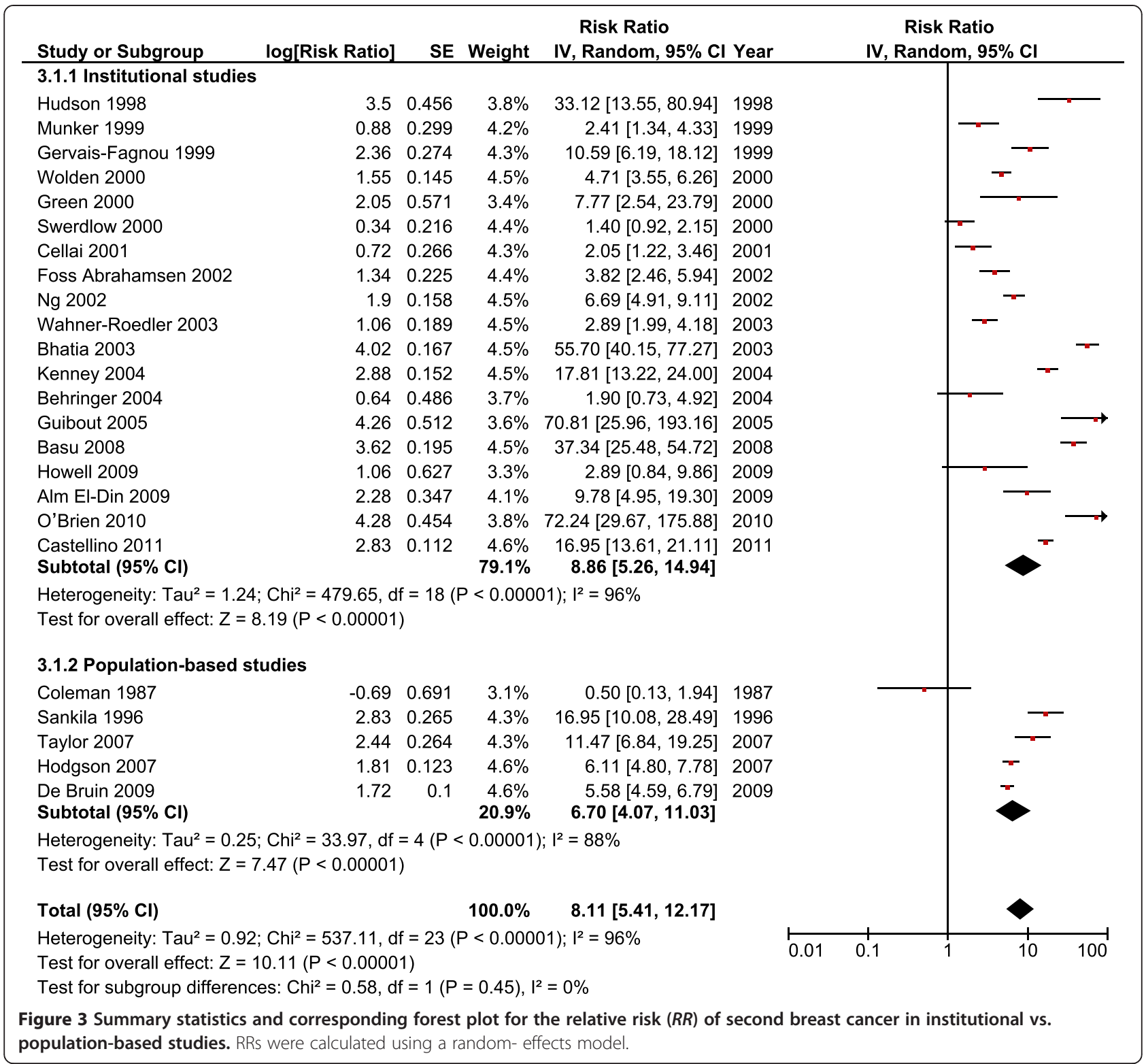

the corresponding RRs for younger and older patients were 14.2 (95\% CI, 7.9-25.4), and 9.0 (95\% CI, 4.9$16.5)$, respectively.

\section{RR and treatment modalities}

Figure 7 shows the random-effects model for the RR according to HL treatment modalities. Significant heterogeneity was demonstrated $\left(I^{2}=87 \%\right)$. RT used as the sole therapeutic modality was associated with an almost 5-fold increase in risk ( $R R=4.70$; [95\% CI, 3.28-6.75]), $\left.I^{2}=74 \%\right)$ and even higher rate $(\mathrm{RR}=14.08$; [95\% CI, 9.93-19.98]) when RT was used for patients $\leq 30$ years of age $[32,36,41]$. Two studies [24,54], reported on the mantle field RT dose where there was a small difference in RR between dose $<40$ Gy and $\geq 40$ Gy (5.99, and 6.13, respectively). In the first study [24], 1 patient per 567 person-years risk versus 23 patients per 7876 personyears developed SBC in the lower versus higher RT dose, respectively. In the second study, 17 of 135 versus 18 of 109 patients developed SBC in the lower versus higher RT dose, respectively [54]. When reported, almost all SBC arose within or at the margin of RT field.

Figure 7 also shows that adding any CT to RT numerically increased the risk as compared with the risk associated with RT only $(\mathrm{RR}=5.65$; [95\%CI, 2.94-10.88], $\left.I^{2}=91 \%\right)$. Nevertheless, adding alkylating CT to RT did not abate SBC risk ( $\mathrm{RR}=6.59$; [95\% CI, 1.72-25.20), while the combination of RT and non-alkylating CT caused a non-significant effect $(\mathrm{RR}=4.40$; [95\% CI, 0.8323.38]) (data not shown). Noteworthy, only a few studies 


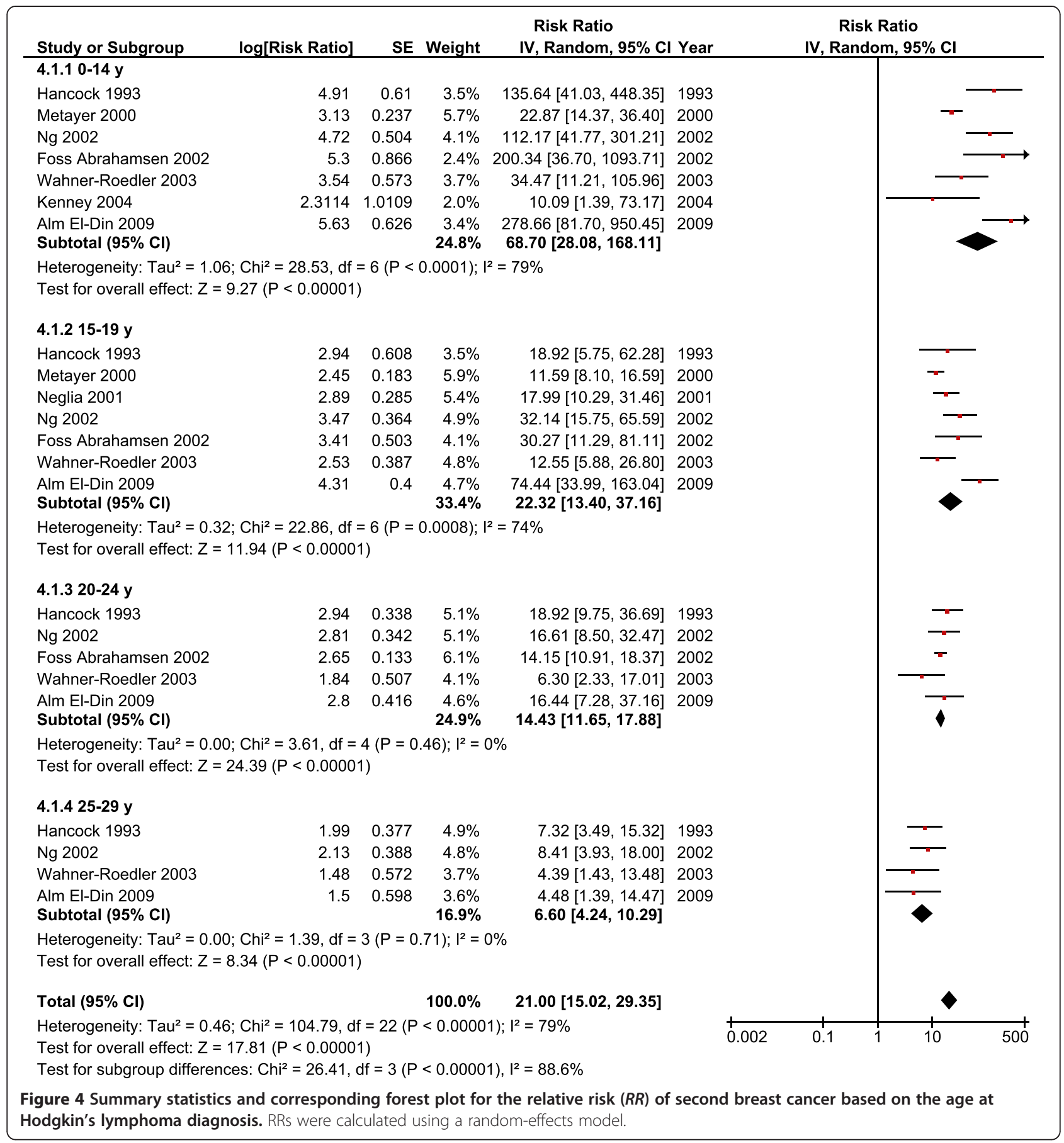

have provided enough data about the nature of CT offered. The current meta-analysis showed that the use of CT only was not associated with significant risk ( $\mathrm{RR}=1.19$; [95\% CI, 0.50-2.82], $I^{2}=87 \%$ ).

To explore the heterogeneity of the RR vs. therapeutic modalities, we performed a univariate analysis that showed an inverse association between $\operatorname{lnRR}$ and age at HL diagnosis, positive interaction with latency, and no significant effect for study size. The multivariate meta-regression analysis, however, only showed that younger age at diagnosis retained a significant independent risk (Table 3).

\section{RR and additional contributing factors}

Comparing HL patients diagnosed from 1960s to the early 1970 s, late 1970 s to early 1980 s, and more recent years the reported RRs were 3.7, 5.9, and 10.7, respectively $[23,28,48,51,55]$. 


\begin{tabular}{|c|c|c|c|c|c|c|c|c|c|}
\hline \multirow{2}{*}{$\begin{array}{l}\text { Study or Subgroup } \\
5.1 .8>20 y\end{array}$} & \multirow[t]{2}{*}{ log[Risk Ratio] } & \multirow[t]{2}{*}{ SE } & \multirow[t]{2}{*}{ Weight } & \multirow[t]{2}{*}{$\begin{array}{l}\text { Risk Ratio } \\
\text { IV, Fixed, } 95 \% \text { CI }\end{array}$} & \multirow[t]{2}{*}{ Year } & \multicolumn{4}{|c|}{$\begin{array}{c}\text { Risk Ratio } \\
\text { IV, Fixed, } 95 \% \mathrm{Cl}\end{array}$} \\
\hline & & & & & & & & & \\
\hline Hancock 1993 & 1.16 & 0.532 & $8.2 \%$ & $3.19[1.12,9.05]$ & 1993 & & & & \\
\hline Foss Abrahamsen 2002 & 1.43 & 0.47 & $10.5 \%$ & $4.18[1.66,10.50]$ & 2002 & & & & \\
\hline $\mathrm{Ng} 2002$ & 1.52 & 0.47 & $10.5 \%$ & $4.57[1.82,11.49]$ & 2002 & & & & \\
\hline De Bruin 2009 & 0.36 & 0.8 & $3.6 \%$ & $1.43[0.30,6.88]$ & 2009 & & & & \\
\hline $\begin{array}{l}\text { Alm El-Din } 2009 \\
\text { Subtotal }(95 \% \mathrm{Cl})\end{array}$ & 1.83 & 0.392 & $\begin{array}{l}15.2 \% \\
\mathbf{4 8 . 1} \%\end{array}$ & $\begin{array}{r}6.23[2.89,13.44] \\
4.26[2.77,6.55]\end{array}$ & 2009 & & & & \\
\hline \multicolumn{10}{|c|}{$\begin{array}{l}\text { Heterogeneity: } \mathrm{Chi}^{2}=3.12, \mathrm{df}=4(\mathrm{P}=0.54) ; \mathrm{I}^{2}=0 \% \\
\text { Test for overall effect: } Z=6.58(P<0.00001)\end{array}$} \\
\hline \multicolumn{10}{|l|}{$5.1 .9>30 y$} \\
\hline Hancock 1993 & -0.41 & 1.271 & $1.4 \%$ & $0.66[0.05,8.01]$ & 1993 & & & & \\
\hline $\mathrm{Ng} 2002$ & 0.58 & 0.8 & $3.6 \%$ & $1.79[0.37,8.57]$ & 2002 & & & & \\
\hline Wahner-Roedler 2003 & 0.18 & 0.378 & $16.3 \%$ & $1.20[0.57,2.51]$ & 2003 & & & & \\
\hline De Bruin 2009 & 0.92 & 0.626 & $5.9 \%$ & $2.51[0.74,8.56]$ & 2009 & & & & \\
\hline $\begin{array}{l}\text { Alm El-Din } 2009 \\
\text { Subtotal }(95 \% \mathrm{Cl})\end{array}$ & 1.53 & 0.326 & $\begin{array}{l}21.9 \% \\
49.3 \%\end{array}$ & $\begin{array}{l}4.62[2.44,8.75] \\
2.42[1.58,3.70]\end{array}$ & 2009 & & & & \\
\hline \multicolumn{10}{|c|}{$\begin{array}{l}\text { Heterogeneity: } \mathrm{Chi}^{2}=8.58, \mathrm{df}=4(P=0.07) ; I^{2}=53 \% \\
\text { Test for overall effect: } Z=4.06(P<0.0001)\end{array}$} \\
\hline \multicolumn{10}{|l|}{$5.1 .10>40 y$} \\
\hline Hancock 1993 & -0.326 & 1.271 & $1.4 \%$ & $0.72[0.06,8.72]$ & 1993 & & & & \\
\hline $\begin{array}{l}\mathrm{Ng} 2002 \\
\text { Subtotal }(95 \% \mathrm{Cl})\end{array}$ & -0.92 & 1.409 & $\begin{array}{l}1.2 \% \\
2.6 \%\end{array}$ & $\begin{array}{l}0.40[0.03,6.31] \\
0.55[0.09,3.52]\end{array}$ & 2002 & & & & \\
\hline \multicolumn{10}{|c|}{$\begin{array}{l}\text { Heterogeneity: } \text { Chi }^{2}=0.10, d f=1(P=0.75) ; l^{2}=0 \% \\
\text { Test for overall effect: } Z=0.63(P=0.53)\end{array}$} \\
\hline Total $(95 \% \mathrm{Cl})$ & & & $100.0 \%$ & $3.05[2.26,4.12]$ & & & & & \\
\hline $\begin{array}{l}\text { Heterogeneity: } \text { Chi }^{2}=18 \\
\text { Test for overall effect: } Z \\
\text { Test for subgroup differe }\end{array}$ & $\begin{array}{l}1, \mathrm{df}=11(P=0.07 \\
7.31(P<0.00001) \\
e s: \mathrm{Chi}^{2}=6.72, \mathrm{df}\end{array}$ & $\begin{array}{l}7) ; I^{2}=4 \\
f=2(P\end{array}$ & $\begin{array}{l}41 \% \\
=0.03), I^{2}\end{array}$ & $=70.2 \%$ & & 0.01 & 0.1 & 10 & 100 \\
\hline
\end{tabular}

Table 4 shows data related to potential contributing factors. Pooled analysis was not attempted due to the small number of studies with sufficient data. Table 4 shows that HL patients who presented with mediastinal mass had higher risk compared with those without mediastinal mass. Table 4 also shows the inconsistency of the reported RR among those who had splenectomy versus those who did no, however, the findings were based on three studies only. Table 4 also shows the potential protective role of pelvic RT as reported by De Bruin et al. [55], where patients receiving that modality showed a risk that was not as high compared with those who did not. HL survivors who received RT had a higher risk of developing estrogen receptor (ER)-negative/progesterone receptor (PR)-negative SBC as compared with ER-positive/PR-positive tumors [57].

\section{Discussion}

HL has been a successful model for the development of effective treatment approach in clinical oncology. Long- term survivors of that disease have also allowed better recognition and understanding of the late effects of therapy. In a large cohort of 25,305 women with HL, and with 957 incidences of SBC reported from North American and European institutions, the current meta-analysis quantified the risk of SBC. The overall pooled analysis showed that there was an approximate 9-fold increase in the risk of $\mathrm{SBC}$ incidence (pooled $\mathrm{RR}=8.23$ ), and $\mathrm{AER}$ of 23 patients per 10,000 person-years.

Patients included in this meta-analysis developed HD at a median age of 23.7 years. There has been incongruity about the influence of younger age at HL diagnosis and the higher risk of SBC, where some studies have failed to prove that effect $[39,42]$. The current meta-analysis clearly showed that younger age at $\mathrm{HL}$ diagnosis was associated with increased risk of SBC, and the risk remained after adjusting for other covariates. Moreover, we demonstrated that the RR of SBC for women who developed HL above the age of 40 years was not significant. It is presumed that the higher risk associated with 


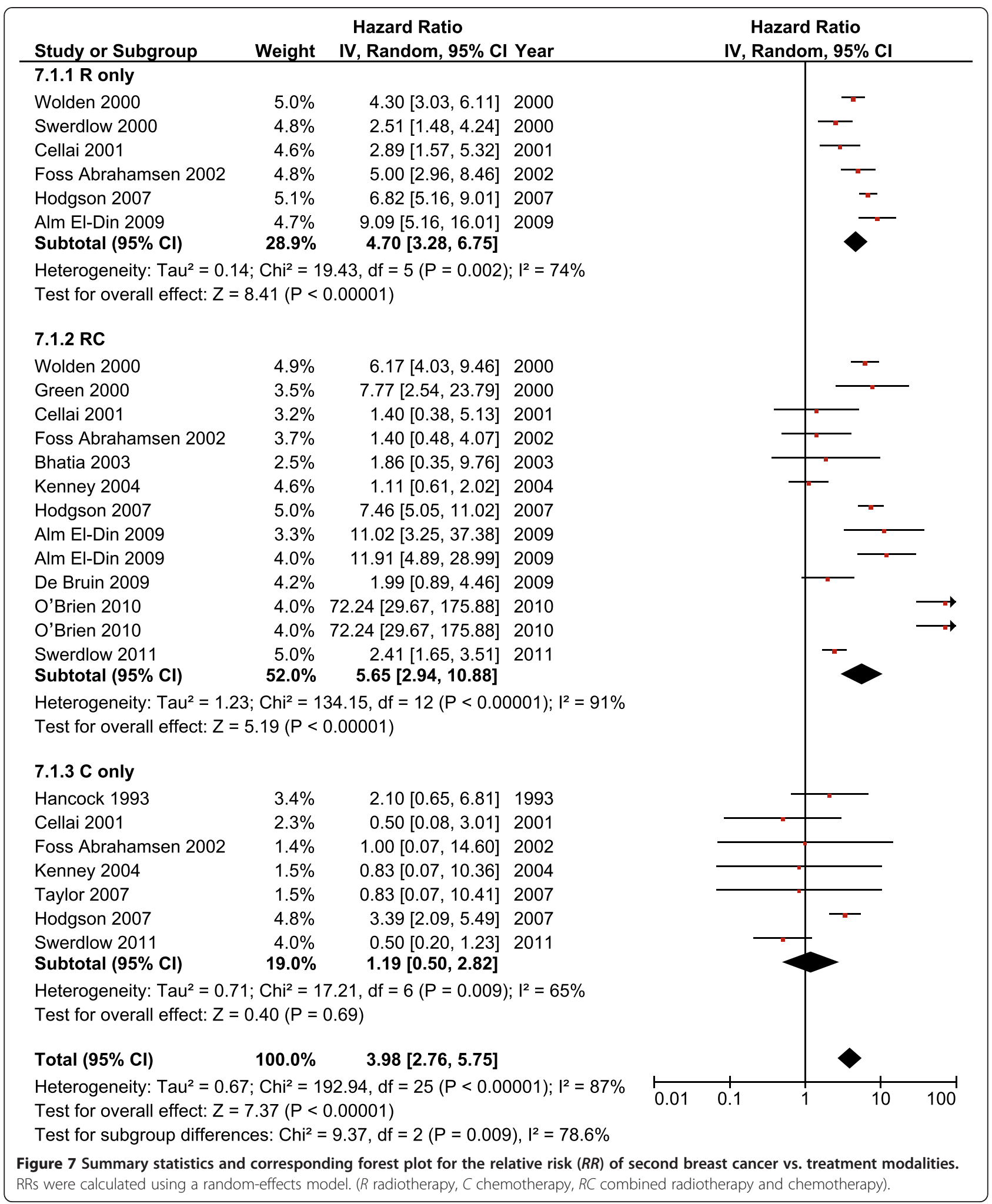

young age at HD diagnosis is attributed to the effect of RT delivered at a time when breast tissue is proliferating.

RT used as the sole therapeutic modality was associated with a 5 -fold $(R R=4.70)$ increase in risk and a 14- fold $(R R=14.08)$ increase among young ( $\leq 30$ years of age). Almost all SBC arose within or at the margin of the RT field. The RR of combined RT and any CT was slightly higher than that associated with RT only (5.65 
Table 4 Relative risk of second breast cancer vs. selected risk variables

\begin{tabular}{|c|c|}
\hline Variable & RR $(95 \% \mathrm{Cl})$ \\
\hline \multicolumn{2}{|l|}{ Mediastinal mass +} \\
\hline Wahner-Roedler 2003 [44] & $4.22(2.71,6.57)$ \\
\hline Alm El-Din 2009 [54] & $11.46(6.78,19.38)$ \\
\hline \multicolumn{2}{|l|}{ Mediastinal mass - } \\
\hline Wahner-Roedler 2003 [44] & $1.70(0.79,3.63)$ \\
\hline Alm El-Din 2009 [54] & $6.09(2.37,15.67)$ \\
\hline \multicolumn{2}{|l|}{ Splenectomy + } \\
\hline Wolden 1998 [30] & $2.10(0.62,7.16)$ \\
\hline Wahner-Roedler 2003 [44] & $4.70(2.87,7.69)$ \\
\hline Alm El-Din 2009 [54] & $9.86(5.42,17.92)$ \\
\hline \multicolumn{2}{|l|}{ Splenectomy - } \\
\hline Wahner-Roedler 2003 [44] & $1.90(1.05,3.45)$ \\
\hline Alm El-Din 2009 [54] & $9.67(4.87,19.20)$ \\
\hline \multicolumn{2}{|l|}{ Mantle RT, no pelvic RT } \\
\hline De Bruin 2009 [55] & $8.20(6.62,10.15)$ \\
\hline \multicolumn{2}{|l|}{ Mediastinal RT, no pelvic RT } \\
\hline De Bruin 2009 [55] & $3.71(1.38,9.97)$ \\
\hline \multicolumn{2}{|l|}{ Mantle RT + pelvic RT } \\
\hline De Bruin 2009 [55] & $2.70(1.11,6.56)$ \\
\hline \multicolumn{2}{|l|}{ ER-/PR- } \\
\hline Dores 2010 [57] & $9.30(7.00,12.36)$ \\
\hline \multicolumn{2}{|l|}{$\mathrm{ER}+/ \mathrm{PR}+$} \\
\hline Dores 2010 [57] & $4.95(3.84,6.39)$ \\
\hline
\end{tabular}

vs. 4.70). An even higher, was the RR associated with the combination of RT and alkylating $\mathrm{CT}(\mathrm{RR}=6.59)$, thus, the potential protective effect of gonado-toxic alkylating CT was not demonstrated. Several studies showed an inverse association between the use of alkylating $\mathrm{CT}$ in $\mathrm{HL}$ and SBC risk [24,65], however, other investigators reported increased risk $[30,66]$. In this meta-analysis and based on data reported from three studies, the risk associated with the combination of RT and non-alkylating CT was not significant $[37,54,58]$, also found insignificant, was the risk related to the use of CT only.

Analysis of potential additional risk factors was limited due to lack of sufficient data and/or inability to compute missing information, therefore, cautions should be exerted in interpreting results. Two studies [24,54], reported on the mantle field RT dose where there was only a small difference in RR between dose $<40$ Gy and $\geq 40$ Gy (5.99, and 6.13, respectively). While some studies showed that subjects with SBC were found to be significantly more likely to have received higher doses of mantle RT [52], this observation was complicated by the fact that patients treated with higher radiation doses have had longer follow-up. Guibout et al. [46], did not find a significant association between RT dose and SBC, suggesting that the increased risk after HL may indicate a specific susceptibility for developing SBC, or a particular susceptibility to radiation and/or chemotherapy, or both. Conversely, De Bruin et al. showed that the risk of $\mathrm{SBC}$ is related to the RT volume [55], where mantle field irradiation was associated with a 2.7 -fold increased risk of SBC compared with mediastinal irradiation alone. Besides, the meta-analysis reported by Franklin et al. showed a RR of 3 comparing extended field versus involved field RT [14].

The reason for failing to show a convincing evidence of RT dose-response effect associated with SBC risk is at best divisive. There is evidence for a strongly linear radiation dose response, but only in the lower dose range (up to 5 or 10 Gy) $[67,68]$. It has been suggested that cell killing tends to decrease the carcinogenic effect of RT along an exponential curve at doses above $10 \mathrm{~Gy}$ [69]. However, it is known that $\mathrm{BC}$ is a known complication of low-dose breast radiation [67], thus $\mathrm{BC}$ may remain a risk among adolescent women who receive any dose of thoracic irradiation for HL.

Although new RT techniques and treatment strategies have the potential to reduce the future burden of late effects, nevertheless, we have shown that an even higher risk was reported in more recent years suggesting that there remains a significant cohort at an increased risk of SBC.

Pelvic RT was found to be associated with a protective effect as reported by De Bruin et al. [55]. The same effect was also noted by Basu et al., where $3.4 \%$ of patients who developed SBC received pelvic RT as compared with $26.3 \%$ among those who did not [52]. It is presumed that the protective effect of pelvic RT is attributed to the induction of premature menopause and the role played by hormone stimulation in RT-induced breast carcinogenesis [43,70]. The influence of splenectomy on SBC risk has been controversial. While some studies reported a modest higher risk [71], other studies failed to show that effect $[30,52]$.

Only one study examined the receptor status of SBC [57]. The RR of ER-negative/PR-negative SBC was $66 \%$ higher than ER-positive/PR-positive SBC among 5-year HL survivors, and nearly two-fold higher among 15-year survivors. Conversely, other studies of small numbers of SBC patients have not found a significant variation in hormone-receptor status when compared with primary $\mathrm{BC}$ controls $[72,73]$. While the incidence of hormone receptor-positive $\mathrm{BC}$ in the general population exceeds that of ER-negative/PR-negative $\mathrm{BC}$, it is postulated, however, that young women treated for HL may experience premature ovarian failure related to HL therapy, 
and therefore, their hormonal BC risk factors may differ from those in the general population.

The present meta-analysis has several limitations. First, it is not possible to completely exclude the possibility that the HL itself carries with it an increased risk of second malignancy including SBC. Second, it is very difficult to quantify the possible effect of confounding factors such as lifestyle factors, personal risk, family history, etc. For example, Landgren et al. found increased RR (1.81) of breast cancer among HL patient with positive (vs. negative) family history of cancer [50]. Third, the analyses showed significant heterogeneity in risk estimate, nevertheless, investigating heterogeneity using meta-regression technique showed the dominant role of age at HL diagnosis. Other limitations include the lack of comprehensive treatment data including information on RT dose and additional treatments, and the lack of sufficient data to model the protective effect of endogenous hormone ablation against the risk associated with exposure to exogenous hormones. Moreover, it is not clear if a similar magnitude of risk is to be expected in a different patient population where the incidence of sporadic BC is low. Finally, the meta-analysis lacks the analysis of SBC outcome. However, SBC incidence rather its mortality was the main objective of the meta-analysis. Moreover, not all studies reported on mortality, besides, analysis of SBC mortality would be confounded by the mortality from HL itself or its therapy-related effect, ascertainment of the cause of death, age of diagnosis of $\mathrm{HL}$ or SBC, and length of follow-up.

\section{Conclusions}

We conclude that the current meta-analysis provided the most recent comprehensive estimate of the risk of SBC in a broad-range of HL survivors with inclusive analysis of relevant clinical and treatment variables. Based on the derived data where the median age at the diagnosis of SBC was 35.0 years and at a median latency of 17.7 years, screening recommendations for HL survivors need to be reemphasized. The results from the current meta-analysis support the favorable outcome of the risk-guided $\mathrm{BC}$ screening for such patients according to three prospective studies [74-76]. It is probably more appropriate that female patients with $\mathrm{HL}$ who are at a higher risk for developing SBC to be screened annually and at an earlier age rather than biennially starting at the age of 50 years as currently recommended for general population [77]. Our data also support the recent trend of risk-adapted management of HL to reduce the risk of short- and long-term adverse events associated with needless overtreatment [78].

\section{Competing interests}

All authors declare that they have no competing interests.

\section{Author details}

'Oncology Center of Excellence, International Medical Center, PO Box 2172, Jeddah 21451, Saudi Arabia. ${ }^{2}$ Princess Noorah Oncology Center, Abdulaziz Medical City, P.O.BOX 9515, Jeddah 21423, Saudi Arabia.

\section{Authors' contributions}

EMI, KMA, GAK, OAA, and MA Conception and design of the meta-analysis. EMI Study coordination and tasks' assignment. KMA, and GAK Initial literature search. EMI, KMA, GAK, OAA, and MA Review of all potential studies. EMI, KMA, GAK, OAA, and MA Data extraction. EMI, KMA, GAK, OAA, and MA Assessing quality of included studies. EMI Statistical analysis. EMI, KMA, and GAK Investigating heterogeneity. EMI, KMA, GAK, OAA, and MA Preparation of the manuscript. EMI, KMA, GAK, OAA, and MA Reading the final manuscript. EMI, KMA, GAK, OAA, and MA Approval of the final manuscript. All authors read and approved the final manuscript.

Received: 2 November 2011 Accepted: 28 May 2012 Published: 28 May 2012

\section{References}

1. Herbst C, Rehan FA, Skoetz N, Bohlius J, Brillant C, Schulz H, Monsef I, Specht $L$, Engert A: Chemotherapy alone versus chemotherapy plus radiotherapy for early stage Hodgkin lymphoma. Cochrane Database Syst Rev 2011, 2:CD007110.

2. Rathore B, Kadin ME: Hodgkin's lymphoma therapy: past, present, and future. Expert Opin Pharmacother 2010, 11(17):2891-2906.

3. Evens AM, Hutchings $M$, Diehl V: Treatment of Hodgkin lymphoma: the past, present, and future. Nat Clin Pract Oncol 2008, 5(9):543-556.

4. Weiner MA, Leventhal B, Brecher ML, Marcus RB, Cantor A, Gieser PW, Ternberg JL, Behm FG, Wharam MD Jr, Chauvenet AR: Randomized study of intensive MOPP-ABVD with or without low-dose total-nodal radiation therapy in the treatment of stages IIB, IIIA2, IIIB, and IV Hodgkin's disease in pediatric patients: a Pediatric Oncology Group study. J Clin Oncol 1997, 15(8):2769-2779.

5. Hodgson DC, Hudson MM, Constine LS: Pediatric hodgkin lymphoma: maximizing efficacy and minimizing toxicity. Semin Radiat Oncol 2007, 17(3):230-242.

6. Canellos GP: Letter: second malignancies complicating Hodgkin's disease in remission. Lancet 1975, 1(7919):1294.

7. Baxi SS, Matasar MJ: State-of-the-art issues in Hodgkin's lymphoma survivorship. Curr Oncol Rep 2010, 12(6):366-373.

8. Miller AB, Howe GR, Sherman GJ, Lindsay JP, Yaffe MJ, Dinner PJ, Risch HA Preston DL: Mortality from breast cancer after irradiation during fluoroscopic examinations in patients being treated for tuberculosis. N Engl J Med 1989, 321(19):1285-1289.

9. Tokunaga M, Land CE, Yamamoto T, Asano M, Tokuoka S, Ezaki H, Nishimori I: Incidence of female breast cancer among atomic bomb survivors, Hiroshima and Nagasaki, 1950-1980. Radiat Res 1987, 112(2):243-272.

10. Neugut Al, Weinberg MD, Ahsan H, Rescigno J: Carcinogenic effects of radiotherapy for breast cancer. Oncology (Williston Park) 1999, 13(9):1261-1245. discussion 1257

11. Wolf J, Schellong G, Diehl V: Breast cancer following treatment of Hodgkin's disease-more reasons for less radiotherapy? Eur J Cancer 1997, 33(14):2293-2294.

12. Rieche K: Carcinogenicity of antineoplastic agents in man. Cancer Treat Rev 1984, 11(1):39-67.

13. Dorr FA, Coltman CA Jr: Second cancers following antineoplastic therapy. Curr Probl Cancer 1985, 9(2):1-43.

14. Franklin J, Pluetschow A, Paus M, Specht $L$, Anselmo AP, Aviles A, Biti G, Bogatyreva T, Bonadonna G, Brillant C, et al: Second malignancy risk associated with treatment of Hodgkin's lymphoma: meta-analysis of the randomised trials. Ann Oncol 2006, 17(12):1749-1760.

15. Henderson TO, Amsterdam A, Bhatia S, Hudson MM, Meadows AT, Neglia JP, Diller LR, Constine LS, Smith RA, Mahoney MC, et al: Systematic review: surveillance for breast cancer in women treated with chest radiation for childhood, adolescent, or young adult cancer. Ann Intern Med 2010, 152 (7):444-455. W144-454

16. Vandenbroucke JP, von Elm E, Altman DG, Gotzsche PC, Mulrow CD, Pocock SJ, Poole C, Schlesselman JJ, Egger M: Strengthening the reporting of observational studies in epidemiology (STROBE): explanation and elaboration. Epidemiology 2007, 18(6):805-835. 
17. Clayton D, Hills M: Statistical Models in Epidemiology. England, Oxford University Press: Oxford; 1993.

18. Alder N, Fenty J, Warren F, Sutton AJ, Rushton L, Jones DR, Abrams KR: Meta-analysis of mortality and cancer incidence among workers in the synthetic rubber-producing industry. Am J Epidemiol 2006, 164(5):405-420.

19. Higgins JP, Thompson SG: Quantifying heterogeneity in a meta-analysis. Stat Med 2002, 21(11):1539-1558.

20. Higgins JP, Thompson SG, Deeks JJ, Altman DG: Measuring inconsistency in meta-analyses. BMJ 2003, 327(7414):557-560.

21. DerSimonian R, Laird N: Meta-analysis in clinical trials. Control Clin Trials 1986, 7(3):177-188

22. Egger M, Davey Smith G, Schneider M, Minder C: Bias in meta-analysis detected by a simple, graphical test. BMJ 1997, 315(7109):629-634

23. Coleman MP, Bell CM, Fraser P: Second primary malignancy after Hodgkin's disease, ovarian cancer and cancer of the testis: a populationbased cohort study. Br J Cancer 1987, 56(3):349-355.

24. Hancock SL, Tucker MA, Hoppe RT: Breast cancer after treatment of Hodgkin's disease. J Natl Cancer Inst 1993, 85(1):25-31.

25. van Leeuwen FE, Klokman WJ, Hagenbeek A, Noyon R, van den BeltDusebout AW, van Kerkhoff EH, van Heerde P, Somers R: Second cancer risk following Hodgkin's disease: a 20-year follow-up study. J Clin Oncol 1994, 12(2):312-325.

26. Mauch PM, Kalish LA, Marcus KC, Coleman CN, Shulman LN, Krill E, Come S, Silver B, Canellos GP, Tarbell NJ: Second malignancies after treatment for laparotomy staged IA-IIIB Hodgkin's disease: long-term analysis of risk factors and outcome. Blood 1996, 87(9):3625-3632.

27. Sankila R, Garwicz S, Olsen JH, Dollner H, Hertz H, Kreuger A, Langmark F, Lanning M, Moller T, Tulinius H: Risk of subsequent malignant neoplasms among 1,641 Hodgkin's disease patients diagnosed in childhood and adolescence: a population-based cohort study in the five Nordic countries. Association of the Nordic Cancer Registries and the Nordic Society of Pediatric Hematology and Oncology. J Clin Oncol 1996, 14(5):1442-1446

28. Aisenberg AC, Finkelstein DM, Doppke KP, Koerner FC, Boivin JF, Willett CG High risk of breast carcinoma after irradiation of young women with Hodgkin's disease. Cancer 1997, 79(6):1203-1210.

29. Hudson MM, Poquette CA, Lee J, Greenwald CA, Shah A, Luo X, Thompson El, Wilimas JA, Kun LE, Crist WM: Increased mortality after successful treatment for Hodgkin's disease. J Clin Oncol 1998, 16(11):3592-3600.

30. Wolden SL, Lamborn KR, Cleary SF, Tate DJ, Donaldson SS: Second cancers following pediatric Hodgkin's disease. J Clin Oncol 1998, 16(2):536-544

31. Gervais-Fagnou DD, Girouard C, Laperriere N, Pintillie M, Goss PE: Breast cancer in women following supradiaphragmatic irradiation for Hodgkin's disease. Oncology 1999, 57(3):224-231.

32. Munker R, Grutzner S, Hiller E, Aydemir U, Enne W, Dietzfelbinger H, Busch M, Haas R, Emmerich B, Schmidt M, et al: Second malignancies after Hodgkin's disease: the Munich experience. Ann Hematol 1999, 78(12):544-554.

33. Garwicz $\mathrm{S}$, Anderson $\mathrm{H}$, Olsen JH, Dollner $\mathrm{H}$, Hertz $H$, Jonmundsson $\mathrm{G}$, Langmark F, Lanning M, Moller T, Sankila R, et al: Second malignant neoplasms after cancer in childhood and adolescence: a populationbased case-control study in the 5 Nordic countries. The Nordic Society for Pediatric Hematology and Oncology. The Association of the Nordic Cancer Registries. Int J Cancer 2000, 88(4):672-678.

34. Green DM, Hyland A, Barcos MP, Reynolds JA, Lee RJ, Hall BC, Zevon MA: Second malignant neoplasms after treatment for Hodgkin's disease in childhood or adolescence. J Clin Oncol 2000, 18(7):1492-1499.

35. Metayer C, Lynch CF, Clarke EA, Glimelius B, Storm H, Pukkala E, Joensuu T, Van Leeuwen FE, Van't Veer MB, Curtis RE, et al: Second cancers among long-term survivors of Hodgkin's disease diagnosed in childhood and adolescence. J Clin Onco 2000, 18(12):2435-2443.

36. Swerdlow AJ, Barber JA, Hudson GV, Cunningham D, Gupta RK, Hancock BW, Horwich A, Lister TA, Linch DC: Risk of second malignancy after Hodgkin's disease in a collaborative British cohort: the relation to age at treatment. J Clin Oncol 2000, 18(3):498-509.

37. Wolden SL, Hancock SL, Carlson RW, Goffinet DR, Jeffrey SS, Hoppe RT Management of breast cancer after Hodgkin's disease. J Clin Oncol 2000, 18(4):765-772

38. Cellai E, Magrini SM, Masala G, Alterini R, Costantini AS, Rigacci L, Olmastroni L, Papi MG, Spediacci MA, Innocenti F, et al: The risk of second malignant tumors and its consequences for the overall survival of Hodgkin's disease patients and for the choice of their treatment at presentation: analysis of a series of 1524 cases consecutively treated at the Florence University Hospital. Int J Radiat Oncol Biol Phys 2001, 49(5):1327-1337.

39. Neglia JP, Friedman DL, Yasui Y, Mertens AC, Hammond S, Stovall M, Donaldson SS, Meadows AT, Robison LL: Second malignant neoplasms in five-year survivors of childhood cancer: childhood cancer survivor study. J Natl Cancer Inst 2001, 93(8):618-629.

40. Foss Abrahamsen A, Andersen A, Nome O, Jacobsen AB, Holte $H$, Foss Abrahamsen J, Kvaloy S: Long-term risk of second malignancy after treatment of Hodgkin's disease: the influence of treatment, age and follow-up time. Ann Oncol 2002, 13(11):1786-1791.

41. Ng AK, Bernardo MV, Weller E, Backstrand K, Silver B, Marcus KC, Tarbell NJ, Stevenson MA, Friedberg JW, Mauch PM: Second malignancy after Hodgkin disease treated with radiation therapy with or without chemotherapy: long-term risks and risk factors. Blood 2002, 100(6):1989-1996.

42. Bhatia S, Yasui Y, Robison LL, Birch JM, Bogue MK, Diller L, DeLaat $C$, Fossati-Bellani F, Morgan E, Oberlin O, et al: High risk of subsequent neoplasms continues with extended follow-up of childhood Hodgkin's disease: report from the Late Effects Study Group. J Clin Oncol 2003, 21(23):4386-4394.

43. Travis LB, Hill DA, Dores GM, Gospodarowicz M, van Leeuwen FE, Holowaty E, Glimelius B, Andersson M, Wiklund T, Lynch CF, et al: Breast cancer following radiotherapy and chemotherapy among young women with Hodgkin disease. JAMA 2003, 290(4):465-475.

44. Wahner-Roedler DL, Nelson DF, Croghan IT, Achenbach SJ, Crowson CS, Hartmann LC, O'Fallon WM: Risk of breast cancer and breast cancer characteristics in women treated with supradiaphragmatic radiation for Hodgkin lymphoma: Mayo Clinic experience. Mayo Clin Proc 2003, 78(6):708-715

45. Kenney LB, Yasui Y, Inskip PD, Hammond S, Neglia JP, Mertens AC, Meadows AT, Friedman D, Robison LL, Diller L: Breast cancer after childhood cancer: a report from the childhood cancer survivor study. Ann Intern Med 2004, 141(8):590-597.

46. Guibout C, Adjadj E, Rubino C, Shamsaldin A, Grimaud E, Hawkins M, Mathieu MC, Oberlin O, Zucker JM, Panis X, et al: Malignant breast tumors after radiotherapy for a first cancer during childhood. J Clin Oncol 2005. 23(1):197-204.

47. Travis LB, Hill D, Dores GM, Gospodarowicz M, van Leeuwen FE, Holowaty E, Glimelius B, Andersson M, Pukkala E, Lynch CF, et al: Cumulative absolute breast cancer risk for young women treated for Hodgkin lymphoma. J Natl Cancer Inst 2005, 97(19):1428-1437.

48. Hodgson DC, Gilbert ES, Dores GM, Schonfeld SJ, Lynch CF, Storm H, Hall P, Langmark $F$, Pukkala $E$, Andersson $M$, et al: Long-term solid cancer risk among 5-year survivors of Hodgkin's lymphoma. J Clin Oncol 2007, 25(12):1489-1497.

49. Koh ES, Tran TH, Heydarian M, Sachs RK, Tsang RW, Brenner DJ, Pintilie M, Xu T, Chung J, Paul N, et al: A comparison of mantle versus involved-field radiotherapy for Hodgkin's lymphoma: reduction in normal tissue dose and second cancer risk. Radiat Oncol 2007, 2:13.

50. Landgren O, Pfeiffer RM, Stewart L, Gridley G, Mellemkjaer L, Hemminki K, Goldin LR, Travis LB: Risk of second malignant neoplasms among lymphoma patients with a family history of cancer. Int J Cancer 2007, 120(5):1099-1102.

51. Taylor AJ, Winter DL, Stiller CA, Murphy M, Hawkins MM: Risk of breast cancer in female survivors of childhood Hodgkin's disease in Britain: a population-based study. Int J Cancer 2007, 120(2):384-391.

52. Basu SK, Schwartz C, Fisher SG, Hudson MM, Tarbell N, Muhs A, Marcus KJ, Mendenhall N, Mauch P, Kun LE, et al: Unilateral and bilateral breast cancer in women surviving pediatric Hodgkin's disease. Int J Radiat Oncol Biol Phys 2008, 72(1):34-40

53. Constine LS, Tarbell N, Hudson MM, Schwartz C, Fisher SG, Muhs AG, Basu SK, Kun LE, Ng A, Mauch P, et al: Subsequent malignancies in children treated for Hodgkin's disease: associations with gender and radiation dose. Int J Radiat Oncol Biol Phys 2008, 72(1):24-33.

54. Alm El-Din MA, Hughes KS, Finkelstein DM, Betts KA, Yock TI, Tarbell NJ, Aisenberg AC, Taghian AG: Breast cancer after treatment of Hodgkin's lymphoma: risk factors that really matter. Int J Radiat Oncol Biol Phys 2009, 73(1):69-74

55. De Bruin ML, Sparidans J, Van't Veer MB, Noordijk EM, Louwman MW, Zijlstra JM, Van Den Berg H, Russell NS, Broeks A, Baaijens MH, et al: Breast 
cancer risk in female survivors of Hodgkin's lymphoma: lower risk after smaller radiation volumes. J Clin Oncol 2009, 27(26):4239-4246.

56. Howell SJ, Searle C, Goode V, Gardener T, Linton K, Cowan RA, Harris MA, Hopwood P, Swindell R, Norman A, et al: The UK national breast cancer screening programme for survivors of Hodgkin lymphoma detects breast cancer at an early stage. Br J Cancer 2009, 101(4):582-588.

57. Dores GM, Anderson WF, Beane Freeman LE, Fraumeni JF Jr, Curtis RE: Risk of breast cancer according to clinicopathologic features among long-term survivors of Hodgkin's lymphoma treated with radiotherapy. Br J Cancer 2010, 103(7):1081-1084.

58. O'Brien MM, Donaldson SS, Balise RR, Whittemore AS, Link MP: Second malignant neoplasms in survivors of pediatric Hodgkin's lymphoma treated with low-dose radiation and chemotherapy. J Clin Oncol 2010, 28(7):1232-1239

59. Castellino SM, Geiger AM, Mertens AC, Leisenring WM, Tooze JA, Goodman P, Stovall M, Robison LL, Hudson MM: Morbidity and mortality in longterm survivors of Hodgkin lymphoma: a report from the Childhood Cancer Survivor Study. Blood 2011, 117(6):1806-1816.

60. Behringer $K$, Josting $A$, Schiller $P$, Eich $H T$, Bredenfeld H, Diehl V, Engert A: Solid tumors in patients treated for Hodgkin's disease: a report from the German Hodgkin Lymphoma Study Group. Ann Oncol 2004, 15(7):1079-1085.

61. Inskip PD, Curtis RE: New malignancies following childhood cancer in the United States, 1973-2002. Int J Cancer 2007, 121(10):2233-2240.

62. Swerdlow AJ, Higgins CD, Smith P, Cunningham D, Hancock BW, Horwich A, Hoskin PJ, Lister TA, Radford JA, Rohatiner AZ, et al: Second cancer risk after chemotherapy for hodgkin's lymphoma: a collaborative british cohort study. J Clin Oncol 2011, 29(31):4096-4104.

63. Janov AJ, Tulecke M, O'Neill A, Lester S, Mauch PM, Harris J, Schnitt SJ, Shapiro CL: Clinical and pathologic features of breast cancers in women treated for Hodgkin's disease: a case-control study. Breast J 2001, 7(1):46-52.

64. Hill DA, Gilbert E, Dores GM, Gospodarowicz M, van Leeuwen FE, Holowaty $\mathrm{E}$, Glimelius B, Andersson M, Wiklund T, Lynch CF, et al: Breast cancer risk following radiotherapy for Hodgkin lymphoma: modification by other risk factors. Blood 2005, 106(10):3358-3365.

65. Tucker MA, Coleman CN, Cox RS, Varghese A, Rosenberg SA: Risk of second cancers after treatment for Hodgkin's disease. N Engl J Med 1988, 318(2):76-81.

66. Kaldor JM, Day NE, Clarke EA, Van Leeuwen FE, Henry-Amar M, Fiorentino MV, Bell J, Pedersen D, Band P, Assouline D, et al: Leukemia following Hodgkin's disease. N Engl J Med 1990, 322(1):7-13.

67. Boice JD Jr: Radiation and breast carcinogenesis. Med Pediatr Oncol 2001 , 36(5):508-513.

68. Preston DL, Mattsson A, Holmberg E, Shore R, Hildreth NG, Boice JD Jr: Radiation effects on breast cancer risk: a pooled analysis of eight cohorts. Radiat Res 2002, 158(2):220-235.

69. Harris JR, Lippman ME, Veronesi U, Willett W: Breast cancer (1). N Engl J Med 1992, 327(5):319-328.

70. Van Leeuwen FE, Klokman WJ, Stovall M, Dahler EC, Van't Veer MB, Noordijk EM, Crommelin MA, Aleman BM, Broeks A, Gospodarowicz M, et al: Roles of radiation dose, chemotherapy, and hormonal factors in breast cancer following Hodgkin's disease. J Natl Cancer Inst 2003, 95(13):971-980.

71. Chung CT, Bogart JA, Adams JF, Sagerman RH, Numann PJ, Tassiopoulos A, Duggan DB: Increased risk of breast cancer in splenectomized patients undergoing radiation therapy for Hodgkin's disease. Int I Radiat Oncol Biol Phys 1997, 37(2):405-409.

72. Gaffney DK, Hemmersmeier J, Holden J, Marshall J, Smith LM, Avizonis V, Tran T, Neuhausen SL: Breast cancer after mantle irradiation for Hodgkin's disease: correlation of clinical, pathologic, and molecular features including loss of heterozygosity at BRCA1 and BRCA2. Int J Radiat Oncol Biol Phys 2001, 49(2):539-546.

73. Castiglioni F, Terenziani M, Carcangiu ML, Miliano R, Aiello P, Bertola L, Triulzi T, Gasparini P, Camerini T, Sozzi G, et al: Radiation effects on development of HER2-positive breast carcinomas. Clin Cancer Res 2007, 13(1):46-51.

74. Diller L, Medeiros Nancarrow C, Shaffer K, Matulonis U, Mauch P, Neuberg D, Tarbell NJ, Litman H, Garber J: Breast cancer screening in women previously treated for Hodgkin's disease: a prospective cohort study. J Clin Oncol 2002, 20(8):2085-2091.
75. Kwong A, Hancock SL, Bloom JR, Pal S, Birdwell RL, Mariscal C, Ikeda DM: Mammographic screening in women at increased risk of breast cancer after treatment of Hodgkin's disease. Breast J 2008, 14(1):39-48.

76. Lee L, Pintilie M, Hodgson DC, Goss PE, Crump M: Screening mammography for young women treated with supradiaphragmatic radiation for Hodgkin's lymphoma. Ann Oncol 2008, 19(1):62-67.

77. Gregory KD, Sawaya GF: Updated recommendations for breast cancer screening. Curr Opin Obstet Gynecol 2010, 22(6):498-505.

78. Hodgson DC: Late effects in the era of modern therapy for Hodgkin lymphoma. Hematology Am Soc Hematol Educ Program 2011, 2011:323-329.

doi:10.1186/1471-2407-12-197

Cite this article as: Ibrahim et al:: Risk of second breast cancer in female Hodgkin's lymphoma survivors: a meta-analysis. BMC Cancer 2012 12:197.

\section{Submit your next manuscript to BioMed Central and take full advantage of:}

- Convenient online submission

- Thorough peer review

- No space constraints or color figure charges

- Immediate publication on acceptance

- Inclusion in PubMed, CAS, Scopus and Google Scholar

- Research which is freely available for redistribution 